\title{
Performance Analysis of Target Depth Classification Algorithm Based on Sea Experiment Data
}

\author{
Anbang Zhao $\mathbb{D}^{1,2,3,4}$ Xuejie Bi, ${ }^{1,2,3}$ Nansong Li $\mathbb{C}^{1,2,3}$ Minghui Zhang, ${ }^{1,2,3}$ \\ and Shengchun Piao $\mathbb{D i}^{1,2,3}$ \\ ${ }^{1}$ Acoustic Science and Technology Laboratory, Harbin Engineering University, Harbin 150001, China \\ ${ }^{2}$ Key Laboratory of Marine Information Acquisition and Security, Harbin Engineering University, Ministry of Industry and \\ Information Technology, Harbin 150001, China \\ ${ }^{3}$ College of Underwater Acoustic Engineering, Harbin Engineering University, Harbin 150001, China \\ ${ }^{4}$ National Key Laboratory of Science and Technology on Underwater Acoustic Antagonizing, China State Shipbuilding Corporation \\ Systems Engineering Research Institute, Beijing 100036, China \\ Correspondence should be addressed to Nansong Li; linansong@hrbeu.edu.cn
}

Received 8 May 2019; Revised 7 August 2019; Accepted 6 September 2019; Published 4 December 2019

Academic Editor: Vincenzo Marletta

Copyright (C) 2019 Anbang Zhao et al. This is an open access article distributed under the Creative Commons Attribution License, which permits unrestricted use, distribution, and reproduction in any medium, provided the original work is properly cited.

\begin{abstract}
Cross-spectrum signals can be calculated by the pressure signals. The sign distribution of cross-spectrum active component can be effectively used for target depth classification algorithm. The algorithm is applicable for depth classification of targets where frequencies can only excite the first two normal modes. The corresponding research results are mainly based on the theoretical study. There are few researches on the algorithm performance based on experiment results. To overcome this research lack, based on the effective depth model, the effects on various receiving depth, source frequency, and received signal-to-noise ratio on the algorithm performance have been studied in this paper. The influence of sound velocity profile parameters (negative gradient, thermocline intensity, thermocline thickness, and up-boundary depth) on the algorithm performance has also been researched. According to the simulation results, proper adjustment of the receiving depths can effectively improve the algorithm performance. The source frequency primarily affects the position of the ideal receiving depth which can be appropriately adjusted according to the depth classification requirements of the real sea environment. The algorithm performance improves gradually with the increase of signal-to-noise ratio. Moreover, the algorithm can also be applied under the conditions of negative gradient and thermocline. The comprehensive sound velocity profile parameters have a large impact on the depth classification performance of the algorithm. Even in the case of strong negative gradient or strong thermocline, the robustness of the algorithm is still high. The feasibility of our presented method has been verified by sea experiment. The practical application value of the ideal receiving depth has been researched and validated. The factors affecting the algorithm performance including line spectrum continuity and received signal-to-noise ratio have also been analyzed in our simulation and real sea experiments.
\end{abstract}

\section{Introduction}

The movement of underwater platforms in channel encounters the security threats caused by various targets. To achieve the passive localization of the target without the target prior information is very essential. The target depth classification results directly related to the target category in the passive localization process are particularly important. Effective classification of targets (surface targets or underwater targets) has a profound impact on the security and concealment of underwater platforms.

The main research focus of this paper is to study a method for target depth classification, which is actually the method for target category classification. In recent years, this area 
of research has developed huge interests and a large number of research work are published in the field of target depth classification.

The initial purpose of the target category is to classify the target depth, rather than accurately estimating the target depth. There are numerous algorithms for depth classification using array signals. Golden [1] performed maximumlikelihood estimation for localization. Liang et al. [2] and Premus et al. [3] proposed mode filtering approaches to acoustic source depth discrimination. Based on either modal decomposition technique [4] or data-based method [5], source depth estimation was realized by Nicolas and Yang. Target depth classification was achieved using a waveguide invariant adaptive matched filter by Goldhahn [6]. There are various researches about target depth estimation on the basis of matched field processing [7-12]. Premus and Backman [13] proposed a matched subspace [14] approach for depth discrimination. The concept of modified modal scintillation index was introduced for source depth classification by An et al. [15]. By using cepstrum techniques, the determination of source depth was carried out by $\mathrm{Wu}$, Zhang et al., and Mitchell and Bedford [16-18]. Source range and depth are obtained using a ray-based backpropagation algorithm by Felisberto et al. [19]. These depth classification algorithms have high accuracy, but most of these methods are not feasible to be applied in real application due to their high complexity and time consumption. In addition, considering the space limitations and security requirements of underwater platforms, it may be impossible to place or tow an array with a large aperture. However, these research hold high theoretical value but need to be further researched for implying in real application scenario for target depth classification by placing one or two sensors.

The cross-spectrum calculation is performed using signals acquired by a single $3 \mathrm{D}$ vector sensor $[20,21]$ or two $2 \mathrm{D}$ vector sensors $[22,23]$, and the target depth classification can be achieved based on the characteristics of the crossspectrum signals. However, most of the above algorithms are theoretical studies [20-23], and the actual performance of the algorithm is rarely analyzed through the analysis of experiment data processing results $[24,25]$. Based on the sea experiment data processing results, this paper analyzes the influence of the ideal receiving depth concept on the performance of the algorithm while verifying the feasibility of the target depth classification algorithm. It shows the real application value of the ideal receiving depth concept. Moreover, the paper makes an in-depth research about the affection of the sound velocity profile parameters (negative gradient, thermocline intensity, thermocline thickness, upboundary depth, etc.) on the algorithm performance.

\section{Theoretical Model}

The acoustic propagation characteristics of shallow water field on the basis of the two-layer fluid-fluid model is shown in Figure 1. $H$ is the sea depth; seawater density $\rho_{1}$ is constant, and the sound velocity of seawater $c_{1}$ is constant or variable with depth $z$ only. The sediment layer is homogeneous fluid half-space whose sound velocity $c_{2}$ and density $\rho_{2}$ are

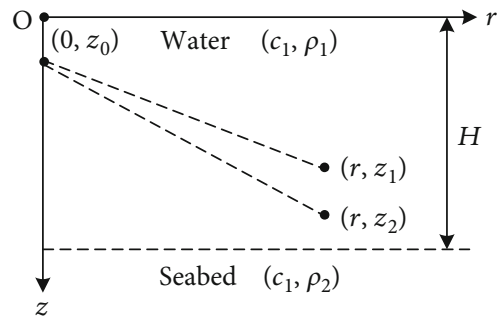

Figure 1: Two-layer fluid-fluid model.

constant. A point source of unit amplitude and angular frequency $\omega$ is located at $\left(0, z_{0}\right)$ in cylindrical coordinates. The receiver is located at $(r, z)$. The sea surface is an absolutely soft interface whose depth is $z=0$ [26].

If $c_{1}$ is constant, this model is known as the Pekeris model. A Pekeris channel of depth $H$ in the shallow water is essentially the same as a waveguide with two pressurerelease boundaries whose depth is $H_{e}$ [27]. $H_{e}$ is called the effective depth.

$$
H_{e}=H\left[1+\frac{1}{b k_{1} H \sin \left(\alpha_{c}\right)}\right]
$$

where $b=\rho_{1} / \rho_{2} ; k_{1}=\omega / c_{1} ; \alpha_{c}=\cos ^{-1}\left(c_{1} / c_{2}\right)$ is the critical grazing angle of the bottom interface. Thus, the pressure field can be expressed as follows:

$$
P\left(z_{0}, z, r\right)=\frac{j}{H_{e}} \sum_{n} \frac{1}{\sqrt{2 \pi \xi_{n} r}} \psi_{n}\left(z_{0}\right) \Psi_{n}(z) e^{-j\left(\xi_{n} r-(\pi / 4)\right)}
$$

where $n$ is the serial number of the normal mode; $\xi_{n}=$ $\sqrt{k_{1}^{2}-\beta_{n}}$ is the $n$th order eigenvalue; $\xi_{n}$ and $\beta_{n}$ are also called horizontal and vertical wave number, respectively. $\Psi_{n}(z)=\sin \left(\beta_{n} z\right)$ is the mode depth function. In the effective depth model, $\beta_{n}=n \gamma, \gamma=\pi / H_{e}$.

$P\left(z_{0}, z_{1}, r\right)$ and $P\left(z_{0}, z_{2}, r\right)$ are signals collected by receiving pressure sensors at different depths $z_{1}$ and $z_{2}$. The active component of their cross-spectrum signal is defined as $I_{p A}$ :

$$
\begin{aligned}
I_{p A} & =\operatorname{Re}\left[P\left(z_{0}, z_{1}, r\right) P^{*}\left(z_{0}, z_{2}, r\right)\right] \\
& =\frac{1}{r}\left[\sum_{n} A_{1 n} A_{2 n}+\sum_{n, n \neq m} \sum_{m} A_{1 n} A_{2 m} \cos \left(\Delta \xi_{m n} r\right)\right] .
\end{aligned}
$$

In the equation, $A_{\text {in }}\left(z_{0}, z_{i}\right)=\left(1 / H_{e} \sqrt{2 \pi \xi_{n}}\right) \Psi_{n}\left(z_{0}\right) \Psi_{n}$ $\left(z_{i}\right), i=1,2$, the superscript ${ }^{*}$ represents the complex conjugate operation; $\operatorname{Re}[\cdot]$ represents the operation of getting real part from the complex number.

The cutoff frequency of the $n$th order normal mode can be calculated as

$$
f_{n}=(2 n-1) \frac{c_{1} c_{2}}{4 H \sqrt{c_{2}^{2}-c_{1}^{2}}}
$$

If source frequency $f$ satisfies $f_{n}<f<f_{n+1}, f$ can excite the first $n$th order normal modes. 


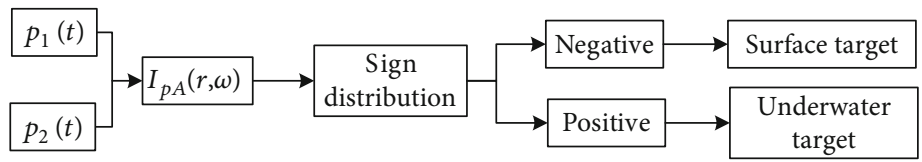

Figure 2: Flow chart of target depth classification. $I_{p A}$ :

If $I_{p A}=1 / r\left(S_{r z}+S_{z}\right), S_{r z}$ and $S_{z}$ are two components of

$$
\begin{aligned}
S_{r z} & =\sum_{n, n \neq m} \sum_{m} A_{1 n} A_{2 m} \cos \left(\Delta \xi_{m n} r\right), \\
S_{z} & =\sum_{n} A_{1 n} A_{2 n} .
\end{aligned}
$$

$S_{r z}$ represents the component of $I_{p A}$ whose sign changes in the horizontal and vertical direction. $S_{z}$ is denoted for the component of $I_{p A}$ whose sign changes in the vertical direction only. According to equation (5), the peak value of $S_{r z}$ is $\sum_{n, n \neq m} \sum_{m} A_{1 n} A_{2 m} . S_{r z}$ resembles cosine signal but its peak value varies with source and receiver depth.

The frequency can only excite the first two normal modes, and target depth classification can be realized using sign distribution of $I_{p A}$. Based on effective depth model, when source frequency can only excite the first two modes, there are ideal receiving depths $z_{1}+z_{2}=H_{e}$ resulting in $\left(A_{11} A_{22}\right.$ $\left.+A_{12} A_{21}\right) \cos \left(\Delta \xi_{12} r\right) \approx 0$, so that $S_{r z}$ is tending to zero. The impact of $S_{r z}$ on the sign distribution of $I_{p A}$ can be ignored. If $z_{1}+z_{2}=H_{e}$, it can guarantee that the sign distribution of $I_{p A}$ will not change in the horizontal direction but change in the vertical direction only.

The critical depth $h_{s}$ is defined as the position of boundary of $I_{p A}$ sign when $z_{1}+z_{2}=H_{e}$. All the signs of $I_{p A}$ above $h_{s}$ are identical, but different from the value lesser than $h_{s}$. The approximate expression of $h_{s}$ is

$$
h_{s} \approx \frac{1}{\gamma} \cos ^{-1}\left[\sqrt{\frac{\xi_{2}}{16 \xi_{1} \cos ^{2}\left(\gamma z_{1}\right)}}\right] .
$$

The flow chart of target depth classification is presented in Figure 2. According to the simulation parameters used in this paper, when the source depth is lesser than $h_{s}$, the sign of $I_{p A}$ is negative, and sources located at these positions can be identified as surface targets. When the source depth is greater than $h_{s}$, the sign of $I_{p A}$ is positive, and the source at these positions can be identified as underwater targets. Corresponding to different simulation parameters, the sign of surface targets' $I_{p A}$ can be positive and the sign of underwater targets' $I_{p A}$ can be negative.

Based on the above theory which have already been published in $[22,23]$, we can conclude that the value of critical depth changes with different receiving depth, source frequency, sound velocity profile, and seabed. The reason why these factors affect algorithm performance has never been researched. We have to develop a model of these parameters affecting the algorithm effectiveness. The selection method of receiving depths has also been described briefly.

Only if $\sqrt{\xi_{2} / 16 \xi_{1} \cos ^{2}\left(\gamma z_{1}\right)} \leq 1$, the existence of critical depth $h_{s}$ can be guaranteed. Thus, the maximum allowable value of the first sensor depth is

$$
z_{1 \max }=\left\lfloor\frac{1}{\gamma} \cos ^{-1}\left(\sqrt{\frac{\xi_{2}}{16 \xi_{1}}}\right)\right\rfloor .
$$

$\lfloor\cdot\rfloor$ represents that fractions are rounded down.

If $z_{1}+z_{2} \neq H_{e}$, the sign of $S_{r z}$ changes in the horizontal direction whose variation law depends on $\cos \left(\Delta \xi_{12} r\right)$ component. The sign of $\cos \left(\Delta \xi_{12} r\right)$ will hop every other $\pi / \Delta \xi_{12}$ in the horizontal direction. The sign of $S_{r z}$ changes in the vertical direction whose variation law depends on $\left(A_{11} A_{22}+\right.$ $\left.A_{12} A_{21}\right)$ component. The sign of $\left(A_{11} A_{22}+A_{12} A_{21}\right)$ will hop at about $H_{e} / 2$. It can be found that whether in the horizontal or vertical direction, the sign hopping of $S_{r z}$ is both not connected with the value of $z_{1}, z_{2}$, but the amplitude of $S_{r z}$ varies with $z_{1}, z_{2}$. So, the sign of $I_{p A}$ will vary with $z_{1}, z_{2}$. At most depths, $S_{r z} \neq 0$, so that $S_{r z}$ will exert significant influence on the sign distribution of $I_{p A}$. On the premise of the existence of the critical depth, the existence of $S_{r z}$ will make the boundary of $I_{p A}$ sign change from straight line to curve that resembles cosine distribution whose peak value changes with $z_{1}, z_{2}$.

Summarizing our presented algorithm, the acoustic field interference structure $I_{p A}$ changes with the variation of receiving depths, source frequency, sound velocity profile, and seabed, which alters the value of $h_{s}$ and the amplitude of $S_{r z}$ accordingly. The change of $h_{s}$ and $S_{r z}$ leads to fluctuate the algorithm performance. The ideal receiving depths corresponding to various conditions are different. So the performance can be improved through proper adjustment of the receiving depths according to the change of these key factors.

\section{Simulation Results}

To verify the feasibility of our presented technique, we conducted a simulation experiment. Sea depth is selected as $H=22 \mathrm{~m}$; densities of seawater and sediment are $\rho_{1}=$ $1.0 \mathrm{~g} / \mathrm{cm}^{3}, \rho_{2}=1.75 \mathrm{~g} / \mathrm{cm}^{3}$; sound velocity in the sediment is $c_{2}=1750 \mathrm{~m} / \mathrm{s}$; source frequency is $f=120 \mathrm{~Hz}$, and the range of source depth is $1 \sim 22 \mathrm{~m}$. The range of horizontal distance is $2 \sim 8 \mathrm{~km} . f_{2}=100 \mathrm{~Hz}, f_{3}=165 \mathrm{~Hz}$; thus, the source frequency can only excite the first two modes. The impact of sound velocity profile on the algorithm performance has been analyzed considering three common types of sound velocity profile (isovelocity, negative gradient, and thermocline) in shallow water. Corresponding to isovelocity profile, sound velocity in the seawater is $c_{1}=1500 \mathrm{~m} / \mathrm{s}$. 

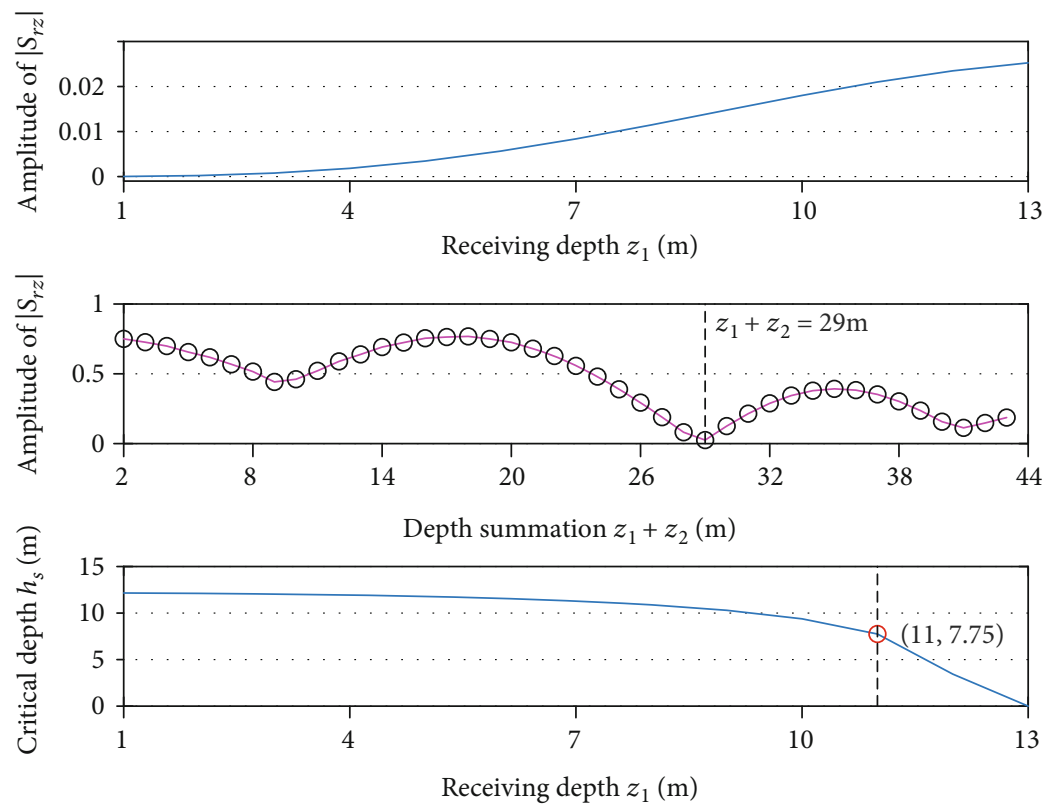

FIGURE 3: Relationship between $S_{r z}, h_{s}$ and $z_{1}, z_{2}$.
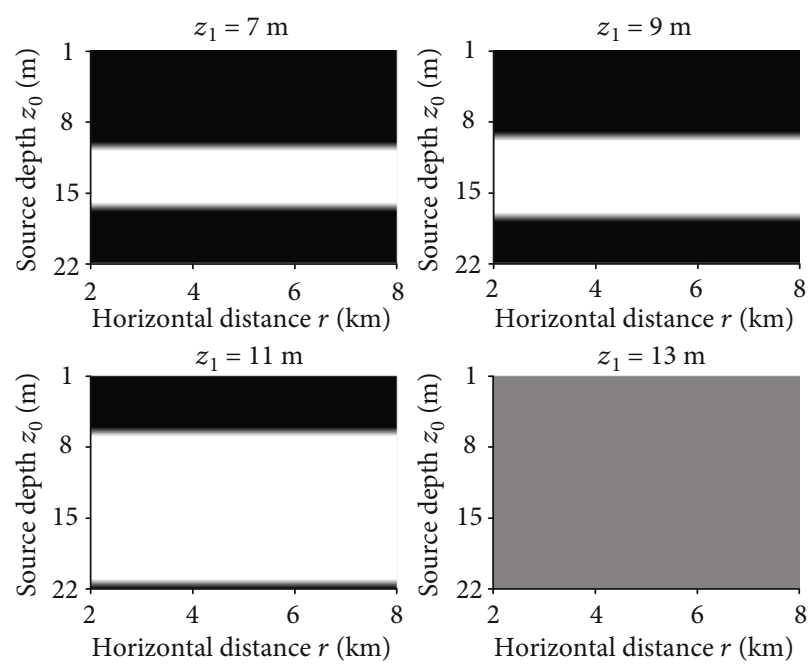

(a)
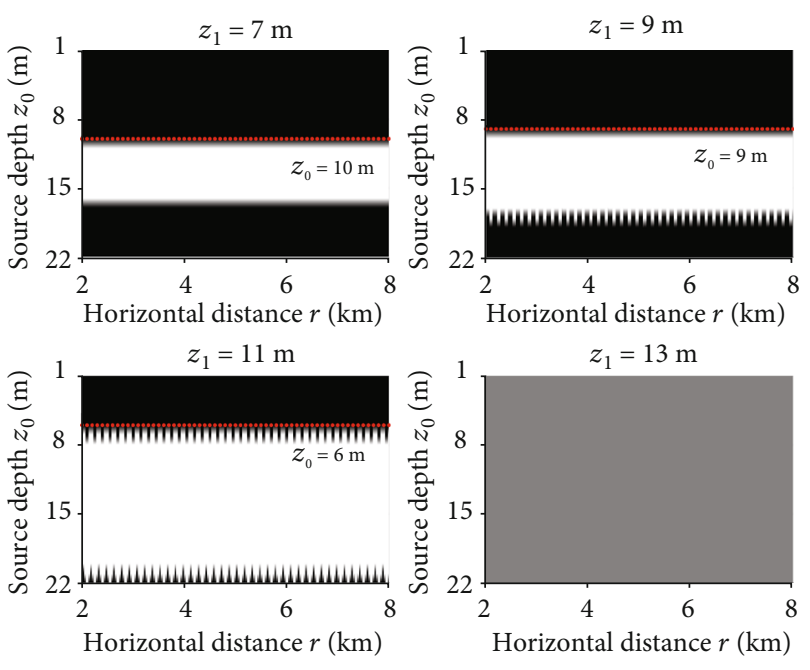

- Critical depth

Figure 4: Impact of $z_{1}$ on the sign distribution of $S_{z}$ and $I_{p A}$ when $z_{1}+z_{2}=28 \mathrm{~m}$. (a) Relationship between $S_{z}$ and $z_{1}$. (b) Relationship between $I_{p A}$ and $z_{1}$.

With respect to different negative gradient profiles, sound velocity in the seawater is $c_{1}=1540 \mathrm{~m} / \mathrm{s}$ at $z=0 \mathrm{~m}$ and $c_{1}=[1520,1500,1480] \mathrm{m} / \mathrm{s}$ at $z=22 \mathrm{~m}$; the gradient is negative and constant, namely, the gradient of different conditions is $G_{c}=[0.9,1.8,2.7] \mathrm{s}^{-1}$.

Thermocline profiles are effected mainly due to thermocline intensity, thermocline thickness, and up-boundary depth. The sound velocity in the seawater is $c_{1}=1540 \mathrm{~m} / \mathrm{s}$ at $z=0 \mathrm{~m}$, and $c_{1}=[1530,1520,1510,1500] \mathrm{m} / \mathrm{s}$ at $z=22 \mathrm{~m}$ corresponding to different conditions. Thus, the thermocline intensity can be $G_{c}=[2.5,5] \mathrm{s}^{-1}$, the thermocline thickness may be considered as $4 \mathrm{~m}, 8 \mathrm{~m}, 12 \mathrm{~m}$, and the up-boundary depth may be $5 \mathrm{~m}, 10 \mathrm{~m}$.

3.1. Impact of Receiving Depth on Algorithm Performance. Under the condition of isovelocity profile, according to equation (1), $H_{e} \approx 29 \mathrm{~m}$. According to equation (8), only if $z_{1} \leq 12 \mathrm{~m}$, target depth classification can be realized. The relationship between $S_{r z}, h_{s}$ and $z_{1}, z_{2}$ is shown in Figure 3. It can be witnessed from Figure 3 that with the increase of $z_{1}$, the peak value of $S_{r z}$ gradually increases. The peak value of $S_{r z}$ will also vary with the distance 

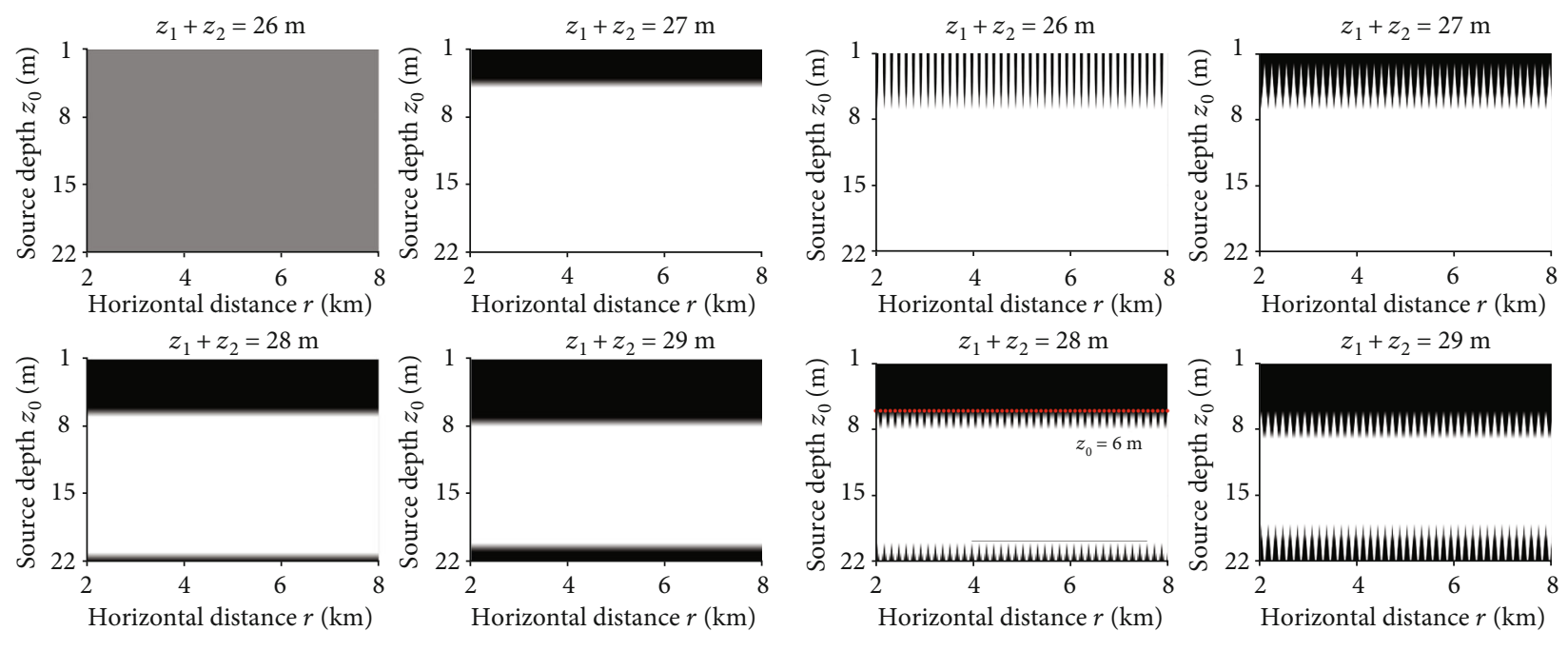

(a)

(b)

FIGURE 5: Impact of $z_{1}+z_{2}$ on the sign distribution of $S_{z}$ and $I_{p A}$ when $z_{1}=11 \mathrm{~m}$. (a) Relationship between $S_{z}$ and $z_{1}+z_{2}$. (b) Relationship between $I_{p A}$ and $z_{1}+z_{2}$.

between $z_{1}+z_{2}$ and $H_{e}$, so as to affect the sign distribution of $I_{p A} ; h_{s}$ will reduce with the increase of $z_{1}$. Consequently, in order to get a better classification of target depth, the value of $z_{1}, z_{2}$ should be considered comprehensively. Firstly, the ideal range of $z_{1}, z_{2}$ should be obtained. Then, conferring to the requirement of $h_{s}$, the ideal range of $z_{1}$ should be confirmed. Finally, in conditions permitting, the value of $z_{1}$ should be reduced appropriately to improve the performance of the depth classification algorithm.

Figure 4 illustrates the impact of $z_{1}$ on the sign distribution of $S_{z}$ and $I_{p A}$. In this article, all sign distributions are indicated by white and grey as positive sign and black indicates negative sign. It can be observed from Figure 4 that the critical depth $h_{s}$ is inversely proportional to $z_{1}$. When $z_{1} \leq 12 \mathrm{~m}$, the target depth classification can be achieved by using the sign distribution of $I_{p A}$. If $z_{1}>12 \mathrm{~m}$, the sign of $I_{p A}$ does not change in the horizontal direction, but the signs are all positive. The sign boundary does not exist and the target depth classification cannot be performed.

Figure 5 expresses the impact of $z_{1}+z_{2}$ on the sign distribution of $S_{z}$ and $I_{p A}$ when $z_{1}=11 \mathrm{~m}$. As it can be witnessed from Figure 5, when $z_{1}+z_{2}=[26,27] \mathrm{m}$, the sign of $I_{p A}$ changes with horizontal distance $r$, which coincides with the change rule shown in Figure 3. With the change of the distance between $z_{1}+z_{2}$ and $H_{e}, S_{r z}$ does not tend to 0. $S_{r z}$ affects the sign distribution of $I_{p A}$ far more than $S_{z}$ resulting in the sign distributions as shown in Figure 5 when $z_{1}+z_{2}$ $=[26,27] \mathrm{m}$. If $z_{1}+z_{2}=[28,29] \mathrm{m}, S_{r z}$ tends to 0 , the sign of $I_{p A}$ hardly changes with $r$. Figure 6 is the sign distribution of $S_{r z}$ under the condition of isovelocity profile. The position of the sign hopping in the vertical direction is about $14 \mathrm{~m}$, roughly equal to $H_{e} / 2$.

3.2. Impact of Source Frequency on Algorithm Performance. When the source frequency can only excite the first two

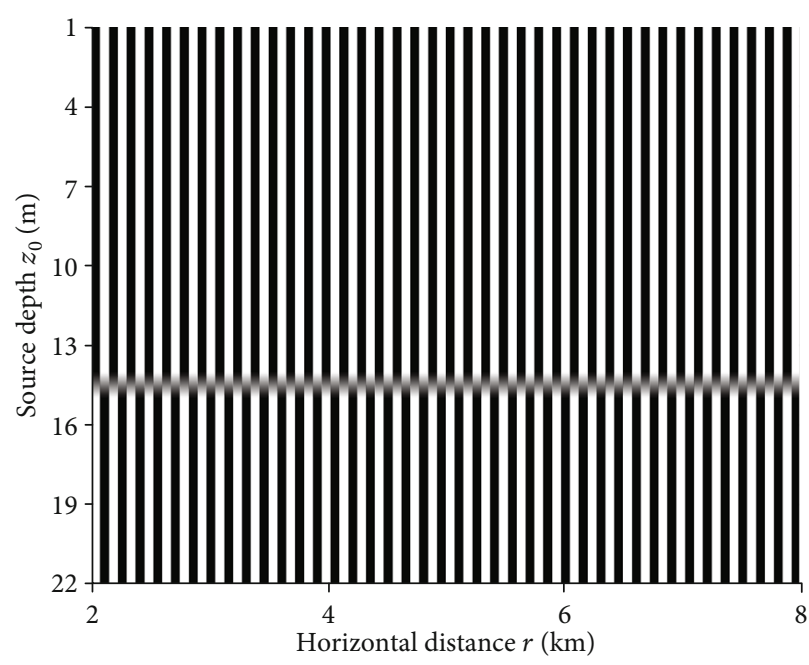

Figure 6: The sign distribution of $S_{r z}$.

modes, the change of the source frequency will affect the target depth classification effect. It can be known from equation (1) that, in case of isovelocity profile, the effective depth $H_{e}$ is inversely proportional to the frequency. The maximum depth of the first receiving sensor $\left(z_{1 \max }\right)$ is related to $H_{e}, \xi_{1}, \xi_{2}$, and the change of the frequency causes the change of the above three variables. Therefore, there is no obvious changing law of $z_{1 \text { max }}$ along with frequency. Similarly, the critical depth $h_{s}$ is also related to $H_{e}, \xi_{1}, \xi_{2}$, so the relationship between $h_{s}$ and frequency does not follow any obvious regular pattern. When $f \in[100,165], H_{e}$ $\epsilon(26.9,30.2)$, the change scope of $H_{e}$ is small. $h_{s}$ and $z_{1 \max }$ are both directly related to $\xi_{2} / \xi_{1}$. When $f \in[100$, $165], \xi_{2} / \xi_{1} \in(0.8875,0.9520)$, the change scope of $\xi_{2} / \xi_{1}$ is also not large. Therefore, as the frequency changes, $h_{s}$ and $z_{1 \max }$ will vary, but the impact of frequency on the 

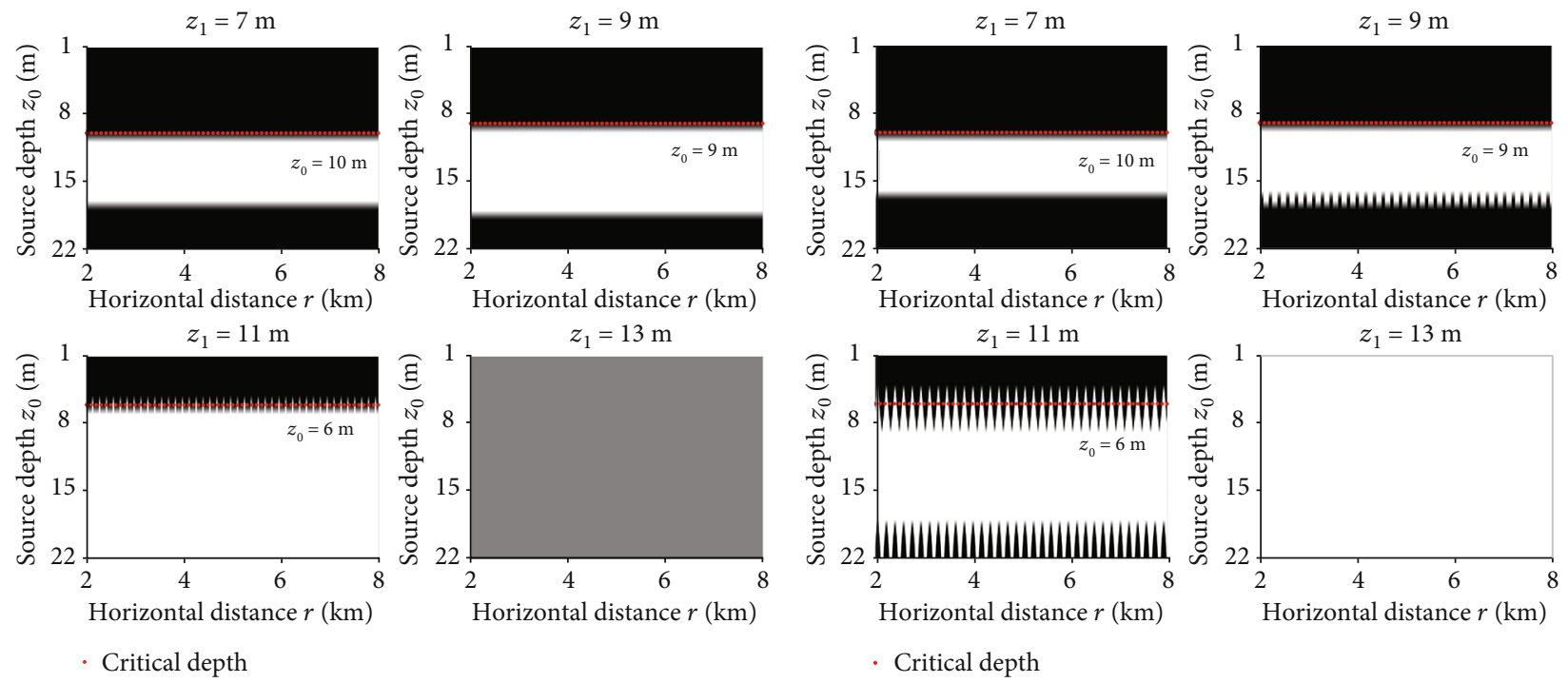

(a)

(b)

Figure 7: The impact of frequency on the sign distribution of $I_{p A}$ under condition of isovelocity profile. (a) $f=110 \mathrm{~Hz}$. (b) $f=150 \mathrm{~Hz}$.

performance of the algorithm is insignificant. When $f=$ $[110,150] \mathrm{Hz}, H_{e}=28 \mathrm{~m}$, the sign distributions of $I_{p A}$ are graphed in Figure 7. Through the analysis of Figure 7, the influence of source frequency on the algorithm performance has been analyzed.

It can be witnessed from Figure 7 that the change of the frequency affects the value of $h_{s}$ and $z_{1 \text { max }}$ and the sign distribution of $I_{p A}$ changes too. However, by adjusting the receiving depth appropriately, based on the sign distribution of $I_{p A}$, the depth classification algorithm can still obtain ideal classification results under different frequency conditions. Therefore, the change in frequency has minor impact on the performance of the algorithm.

\subsection{Impact of Signal-Noise Ratio on Algorithm Performance.} In this section, we will discuss the effect of SNR on the performance of our presented algorithm. The research object changes from point source to radiation noise of surface or underwater targets. The parameters considered are as follows: heading angle is $\psi=50^{\circ}$, target velocity is $v_{t}=11 \mathrm{~m} / \mathrm{s}$, closest distance is $r_{\min }=1200 \mathrm{~m}$, initial distance is $r_{0}=2000$ $\mathrm{m}$, platform velocity is $v_{s}=2 \mathrm{~m} / \mathrm{s}$, and range of SNR (signalnoise ratio) is $\mathrm{SNR}=-30 \sim 0 \mathrm{~dB}$. Assuming that the target radiated noise is a single-frequency line spectrum superimposed on the continuum, two pressure sensors are used to receive signals at depths of $45 \mathrm{~m}$ and $90 \mathrm{~m}$, respectively, and the total sailing time is $400 \mathrm{~s}$. The target is kept in motion away from the receiving platform. With respect to the abovementioned simulation parameters, the influence of SNR on the performance of the algorithm is analyzed. Figure 8 expresses the relationship between the performance of the target depth classification algorithm and SNR under isovelocity condition. In the process of target depth classification, the index that implies the performance of the algorithm is the probability of identified as underwater target in percent-

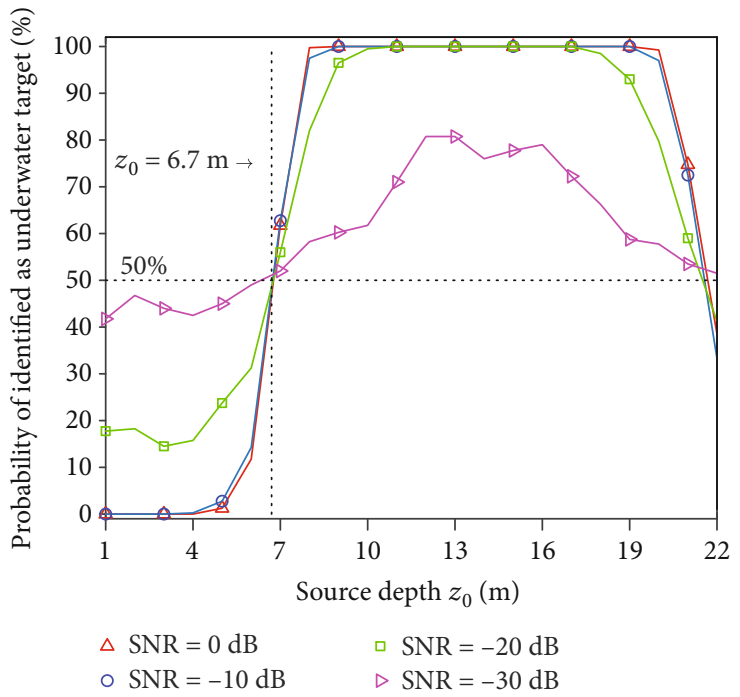

FIGURE 8: The impact of SNR on the algorithm performance under condition of isovelocity profile.

age. The probability of identified as underwater target is defined as the proportion of the cases in which the sign of the pressure cross-spectrum active component is positive. It is equal to $\sum_{i=1}^{N_{1}} L_{i} / M_{1} N_{1} \times 100 \%$. $N_{1}$ is the total number of times of Monte Carlo simulation; $M_{1}$ is the total sampling numbers in each Monte Carlo simulation; $L_{i}\left(i=1,2, \cdots, N_{1}\right)$ is the number of times of the cases in which the sign is positive when discriminating the sign of the pressure cross-spectrum active component in each simulation, $0 \leq L_{i} \leq M_{1}$. It can be found from Figure 8 that when $S N R \geq-20 \mathrm{~dB}$, the target depth classification effect is good, and there is an obvious sign boundary at about $7 \mathrm{~m}$. When $\mathrm{SNR}=-30 \mathrm{~dB}$, the algorithm can achieve the target depth classification, but the classification effect is poor. 


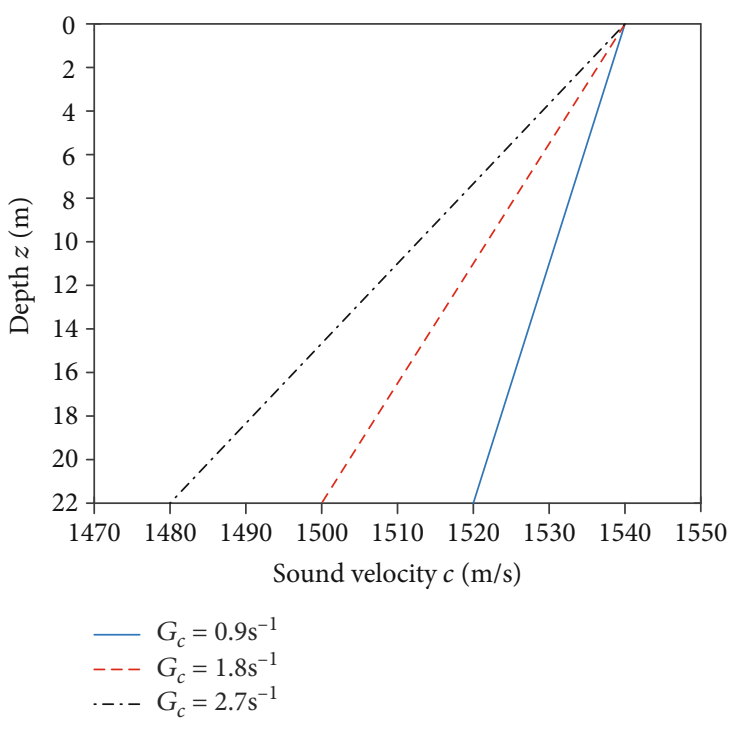

(a)
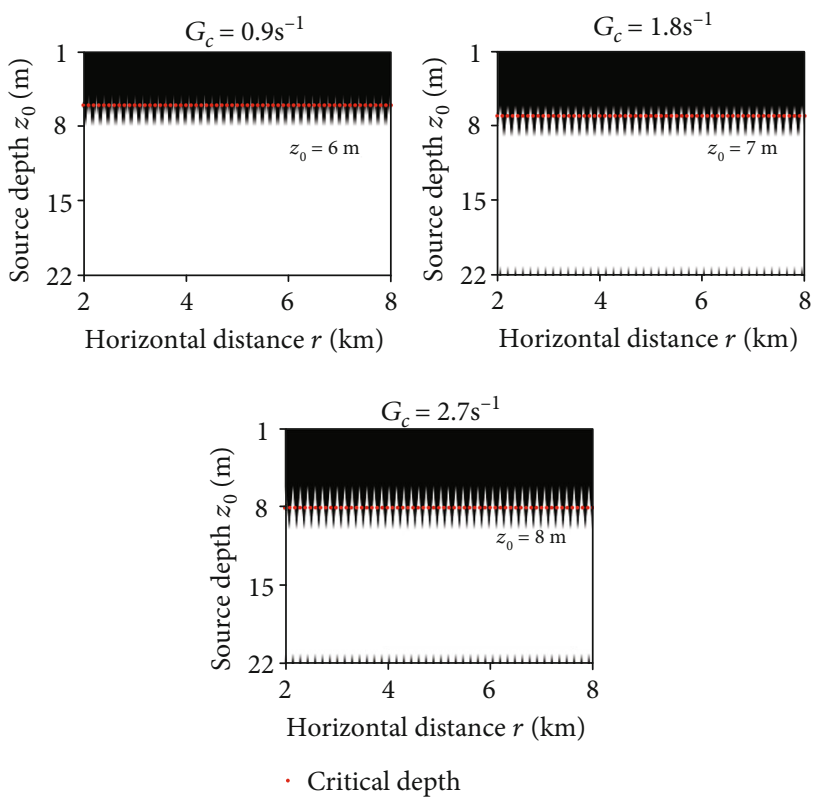

(b)

FIgURE 9: The impact of gradient on the algorithm performance under condition of negative gradient sound velocity profile. (a) Sound velocity profile. (b) The sign distribution of $I_{p A}$.

\subsection{Impact of Sound Velocity Profile on Algorithm Performance}

3.4.1. Negative Gradient Sound Velocity Profile. Receiving depths are considered as $z_{1}=11 \mathrm{~m}, z_{2}=17 \mathrm{~m}$. The negative gradient sound velocity profiles corresponding to different negative gradient $G_{c}=[0.9,1.8,2.7] \mathrm{s}^{-1}$ are shown in Figure 9(a). Under different conditions of $G_{c}=[0.9,1.8,2.7]$ $\mathrm{s}^{-1}$, the sign distributions of $I_{p A}$ are shown in Figure 9(b). It can be witnessed from Figure 9 that the sound velocity gradient is inversely proportional to the depth classification effect and is directly proportional to the critical depth. Even in the case of strong negative gradient, the robustness of the algorithm is high.

3.4.2. Thermocline Existing in Sound Velocity Profile. When the thermocline thickness is fixed to $8 \mathrm{~m}$ and the upboundary depth is fixed to $5 \mathrm{~m}$, the sound velocity profiles and the sign distributions of $I_{p A}$ under different conditions of $G_{c}=[2.5,5] \mathrm{s}^{-1}$ are displayed in Figure 10. When the thermocline intensity is fixed to $G_{c}=2.5 \mathrm{~s}^{-1}$ and the thermocline thickness is fixed to $8 \mathrm{~m}$, the sound velocity profiles and the sign distributions of $I_{p A}$ when the up-boundary depths are $5 \mathrm{~m}, 10 \mathrm{~m}$ are shown in Figure 10.

It can be witnessed from Figure 10 that the algorithm in this paper is also applicable when thermocline exists in the sound velocity profile. The depth classification effect of the algorithm deteriorates as thermocline intensity increases, and the critical depth value is nearly constant. While the depth classification effect of the algorithm deteriorates as the depth of the up-boundary increases, the critical depth value almost keeps the same. In the presence of thermocline, both the thermocline intensity and up-boundary depth have a large impact on the depth classification performance of the algorithm, but the critical depth value remains almost the same. Even with the presence of a strong thermocline, the depth classification performance of the algorithm is good.

When the thermocline intensity is fixed to $G_{c}=2.5 \mathrm{~s}^{-1}$ and the up-boundary depth is fixed to $5 \mathrm{~m}$, the sound velocity profiles and the sign distributions of $I_{p A}$ when the thermocline thicknesses are $4 \mathrm{~m}, 8 \mathrm{~m}, 12 \mathrm{~m}$ are displayed in Figure 11.

It can be seen from Figure 11 that the depth classification effect of the algorithm deteriorates with the increase of the thermocline thickness and the critical depth value increases with the increase of the thermocline thickness. Therefore, the thermocline thickness has a great impact on the depth classification performance of the algorithm.

\section{Experiment Results}

In order to examine the influence of the ideal receiving depth concept on the performance of target depth classification algorithm, an experiment was carried out in Yellow Sea in Qingdao, China. The layout of the sea trial is displayed in Figure 12. The source is suspended into the water by the sending ship at about $8 \mathrm{~m}$. The sound velocity profile near the source position is calculated by CTD (Conductivity-Temperature-Depth System). The CTD device is shown in Figure 13(a), and the collected sound velocity profile is graphed in Figure 14. It can be witnessed from Figure 14 that 


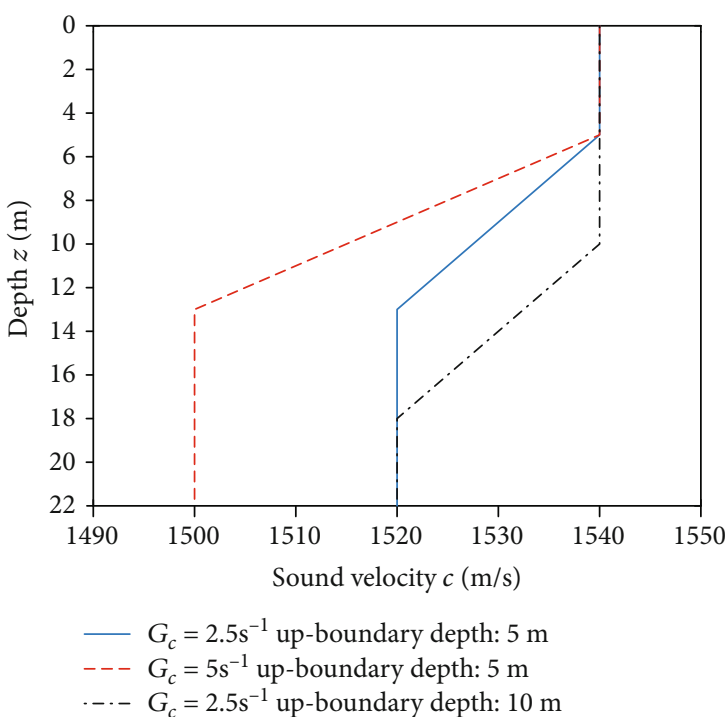

(a)
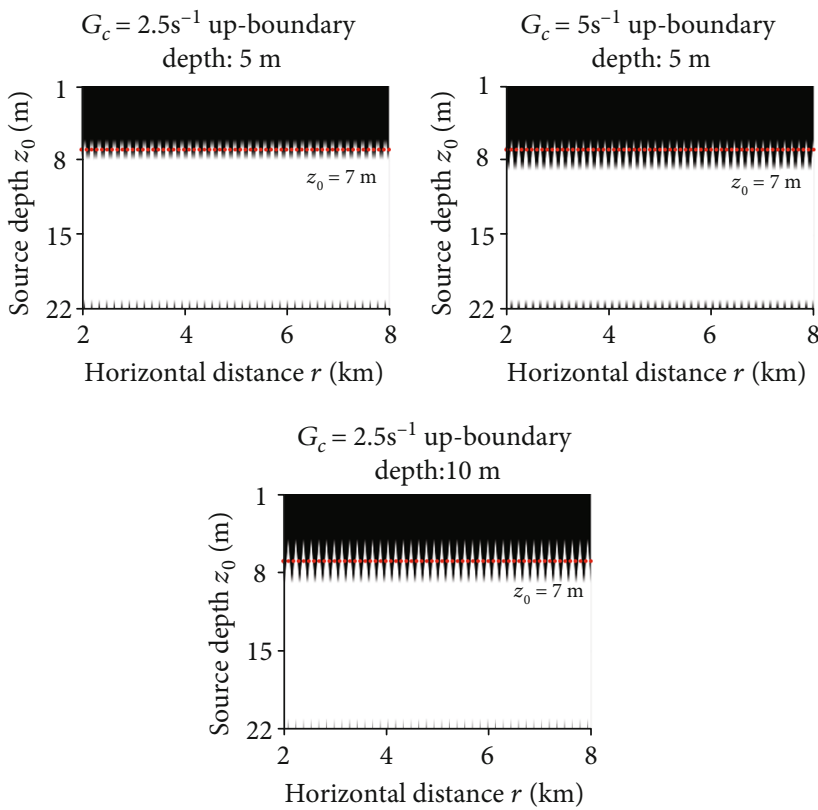

Critical depth

(b)

FIGURE 10: The impact of thermocline intensity and up-boundary depth on the algorithm performance under condition of thermocline existing in profile. (a) Sound velocity profile. (b) The sign distribution of $I_{p A}$.

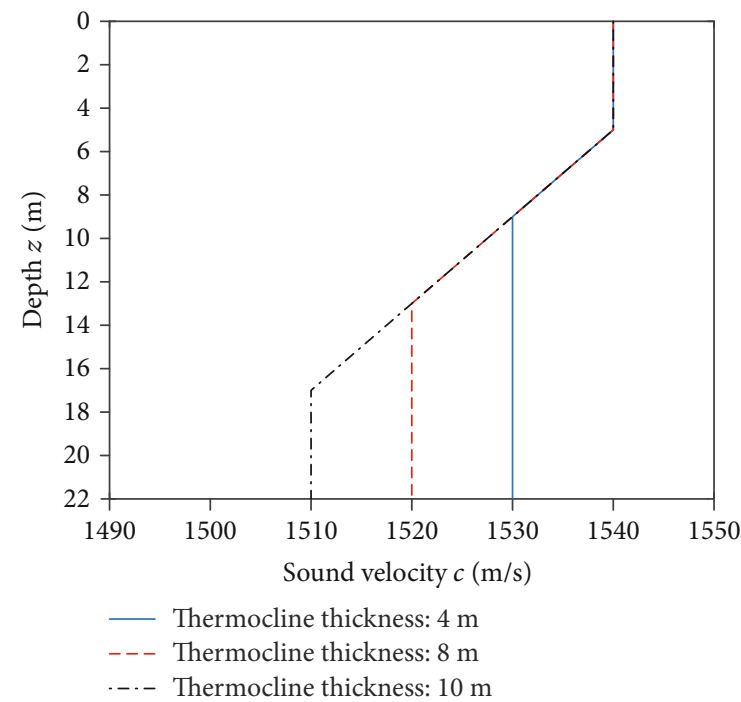

(a)
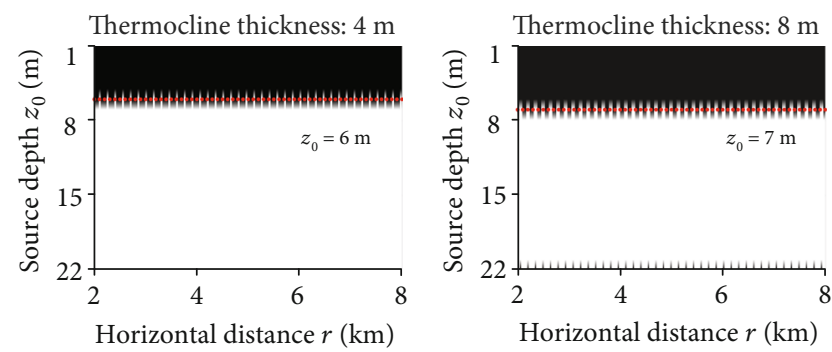

Thermocline thickness: $10 \mathrm{~m}$

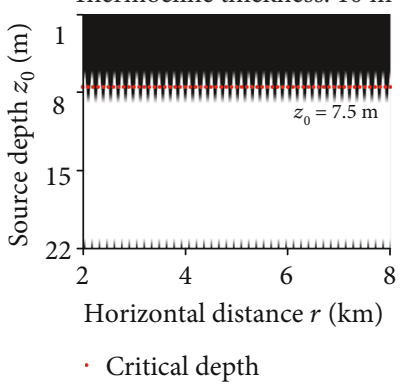

(b)

FIGURE 11: The impact of thermocline thickness on the algorithm performance under condition of thermocline existing in profile. (a) Sound velocity profile. (b) The sign distribution of $I_{p A}$. 


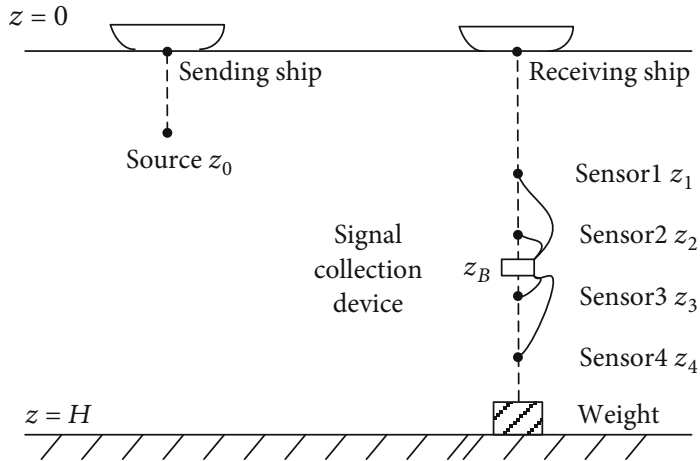

Figure 12: Sea experiment layout diagram.

the type of the sound velocity profile is negative gradient sound velocity profile.

Four receiving pressure sensors are equally spaced into the water by the receiving ship, and one TD (depth sensor) is fixed at each sensor. The signal collection device is fixed between the second and third sensors, and all sensors are connected to the signal collection device. The effect of the ocean current on the experiment results is reduced by fixing the weight on the rope. The receiving device is shown in Figure 13(b).

According to the CTD, the sea depth is approximately $22 \mathrm{~m}$. It can be obtained from equation (1) that $H_{e} \in(29,30$ )m. According to equation (8), $z_{1 \text { max }}<13 \mathrm{~m}$. The length of the pressure sensor cable is limited; in order to ensure the stability of the sensor power supply, the overall length of pressure array length cannot exceed $6 \mathrm{~m}$. The four array elements are laid at equal intervals to keep the interval between two neighboring elements of $2 \mathrm{~m}$. The designed depths of the four array elements are $11,13,15$, and $17 \mathrm{~m}$. According to the TD record, the actual depths of the four elements are $\left[z_{1}, z_{2}, z_{3}, z_{4}\right] \approx[10.8,12.5,14.6,16.5] \mathrm{m}$. Due to the existence of the sea current in the sea, the four elements are deviated slightly from their designed depth. When the sending ship is about 3,5 , and $7 \mathrm{~km}$ away from the receiving ship, the single-frequency continuous sine transmitted by the source is repeated for two times where its frequency is $120 \mathrm{~Hz}$ and its time length is $1 \mathrm{~min}$. The serial number of the corresponding signals is signal 1 to signal 6 . As there are a total of four array elements, during the processing of the transmitted signal, signals collected by two elements are selected to perform the target depth classification using the algorithm described in this paper. There are six different combinations of elements. Different combinations of elements can result in different target depth classification results. A sort is defined for the six element combinations, and the serial number of element combinations is 1 to 6 , corresponding to $\left(z_{1}, z_{2}\right)$, ( $\left.z_{1}, z_{3}\right),\left(z_{1}, z_{4}\right),\left(z_{2}, z_{3}\right),\left(z_{2}, z_{4}\right)$, and $\left(z_{3}, z_{4}\right)$, respectively.

If the parameters of the negative gradient sound velocity profile in the experiment are approximated as $c_{1}=1540 \mathrm{~m} / \mathrm{s}$ at $z=0 \mathrm{~m}$ and $c_{1}=1520 \mathrm{~m} / \mathrm{s}$ at $z=22 \mathrm{~m}$, the simulation analyzes the effect of receiving depths on depth classification performance under negative gradient conditions, as shown in Figure 15.

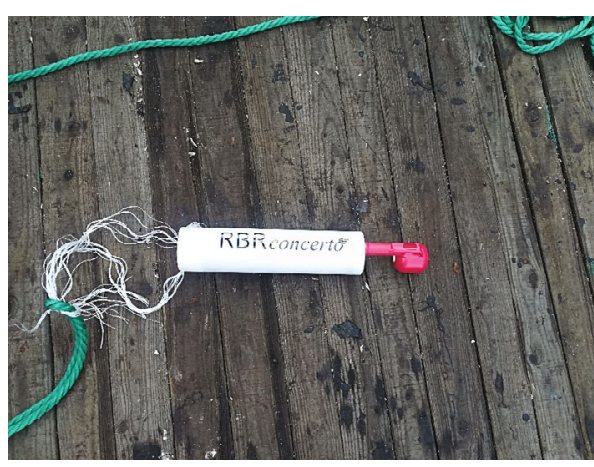

(a)

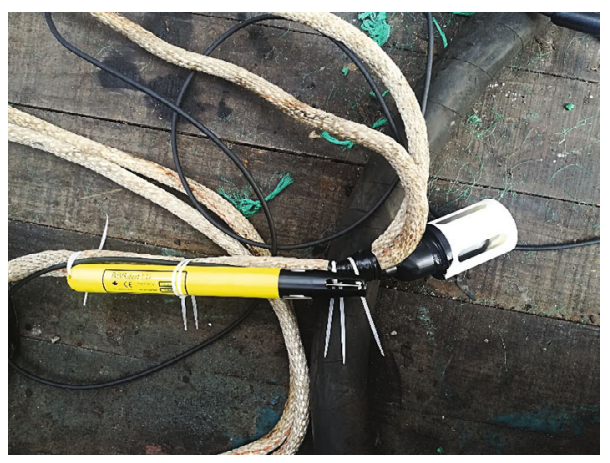

(b)

FIgURE 13: Devices used in the sea experiment. (a) CTD. (b) Receiving sensor and TD.

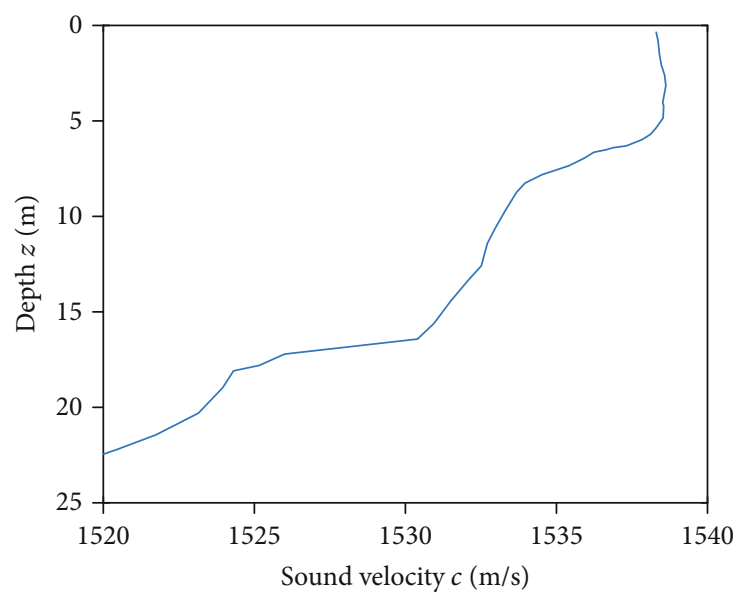

FIGURE 14: Sound velocity profile.

The algorithm presented in this paper is applicable to the isovelocity condition as well as to the negative gradient condition. Based on the signals collected by the third and fifth element combinations, the sign distributions of the calculated $I_{p A}$ can be used for depth classification of both surface targets and underwater targets. The other four combinations can only accurately classify the depth of the underwater targets but cannot accurately classify the depth of the surface targets. In addition, although the critical depth of the fifth combination is smaller than that of the third combination, the sign boundary of the third combination has a smaller 


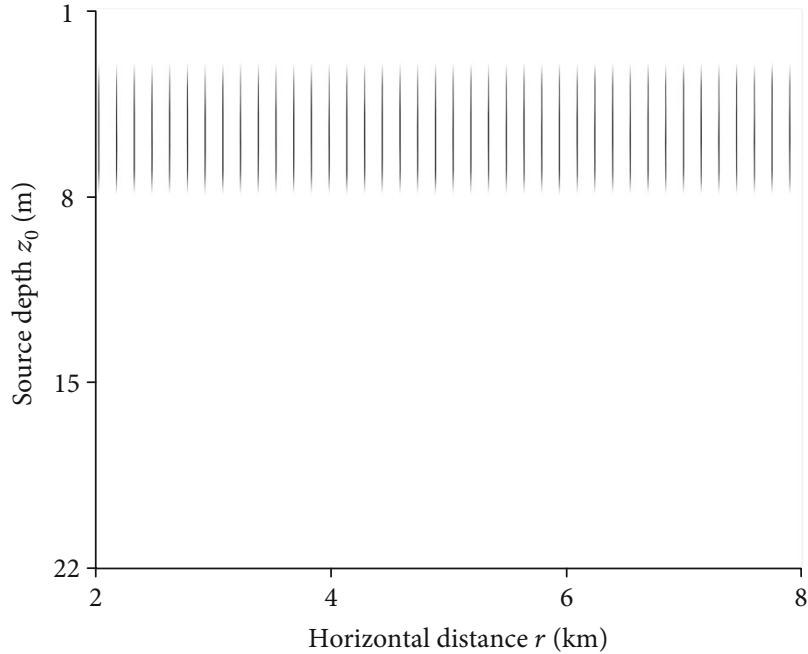

(a)

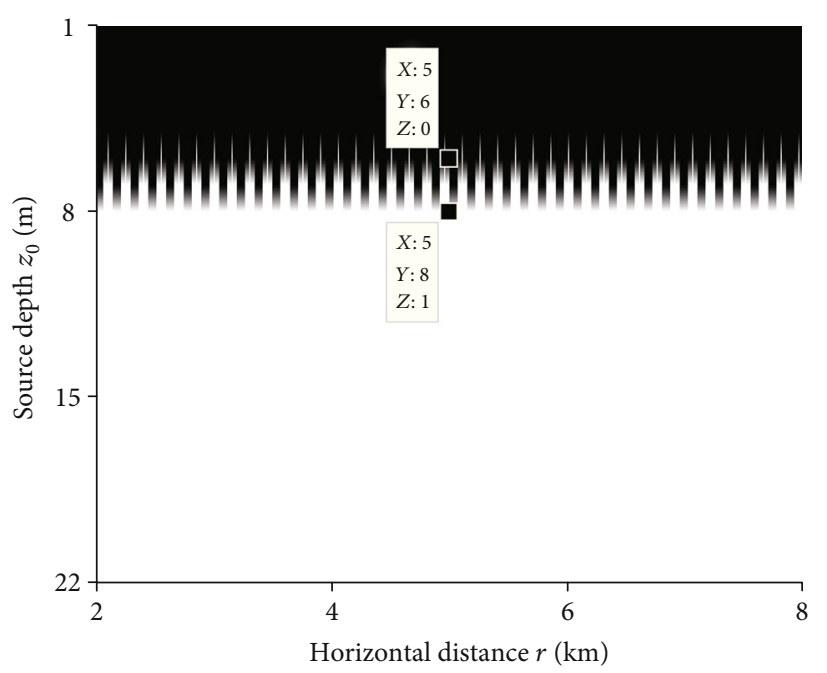

(c)

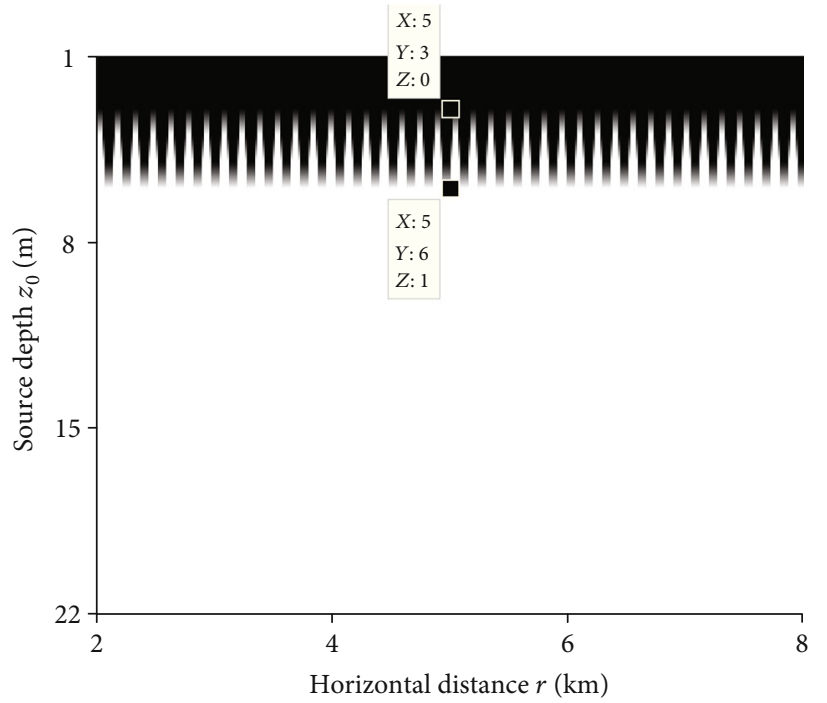

(e)

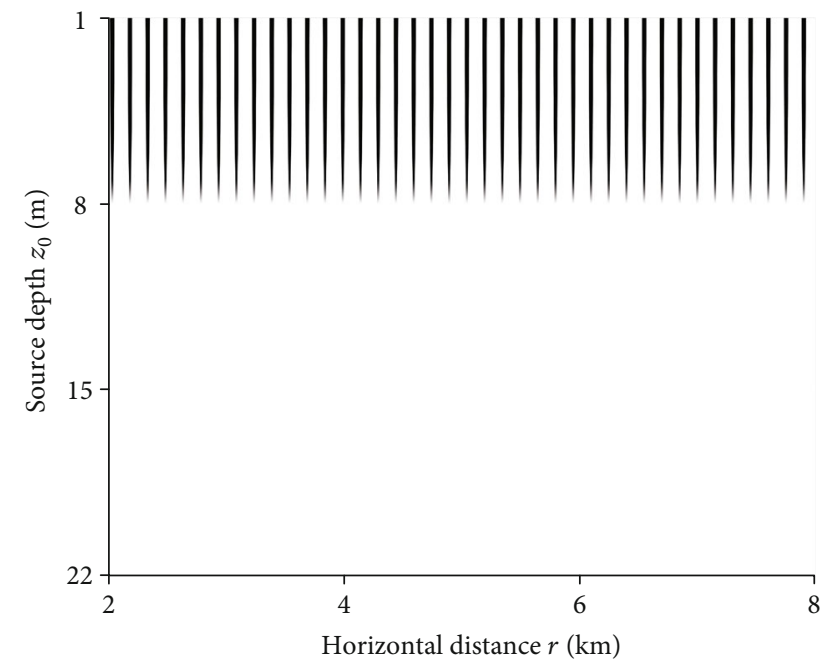

(b)

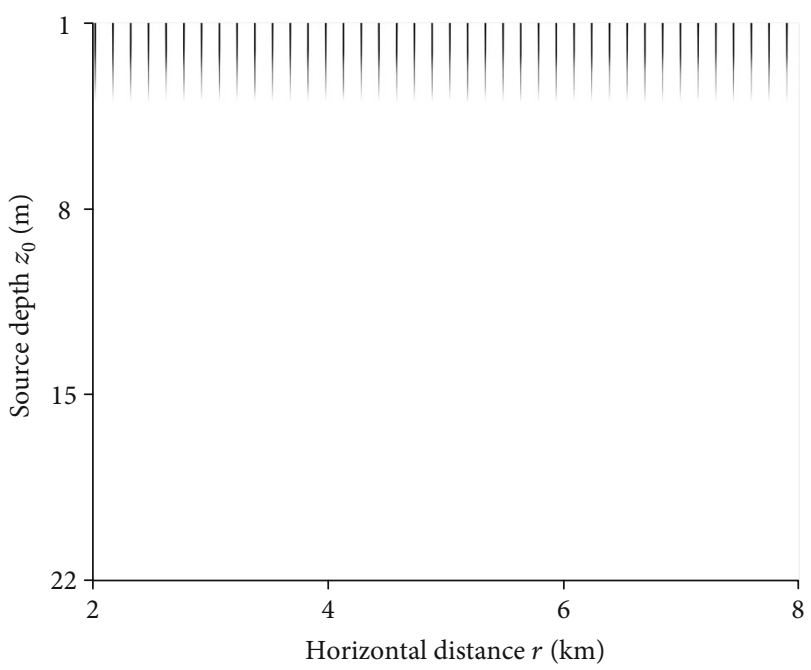

(d)

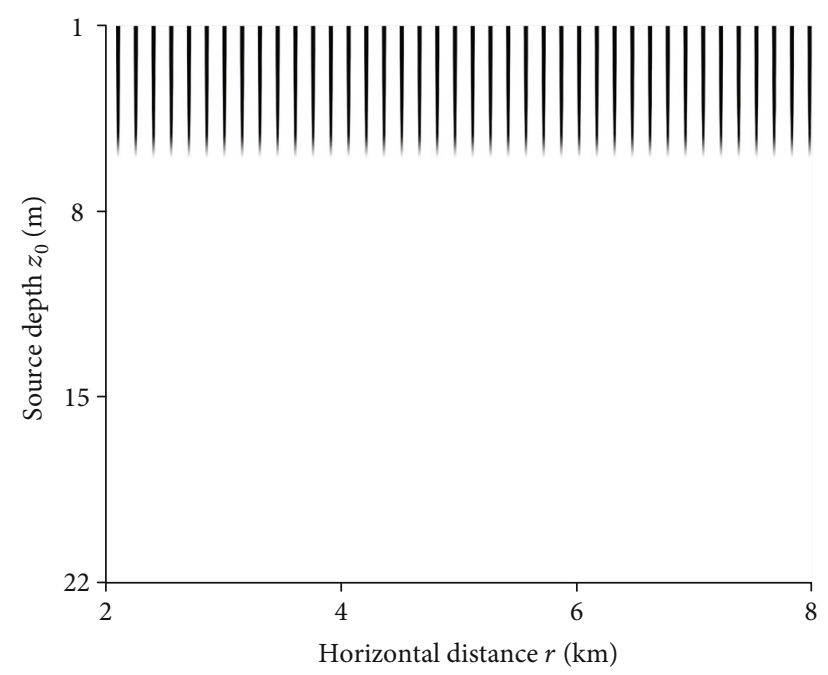

(f)

FIGURE 15: Relationship between the effect of depth classification and receiving depths under the condition of negative gradient profile. (a) First element combination. (b) Second element combination. (c) Third element combination. (d) Fourth element combination. (e) Fifth element combination. (f) Sixth element combination. 

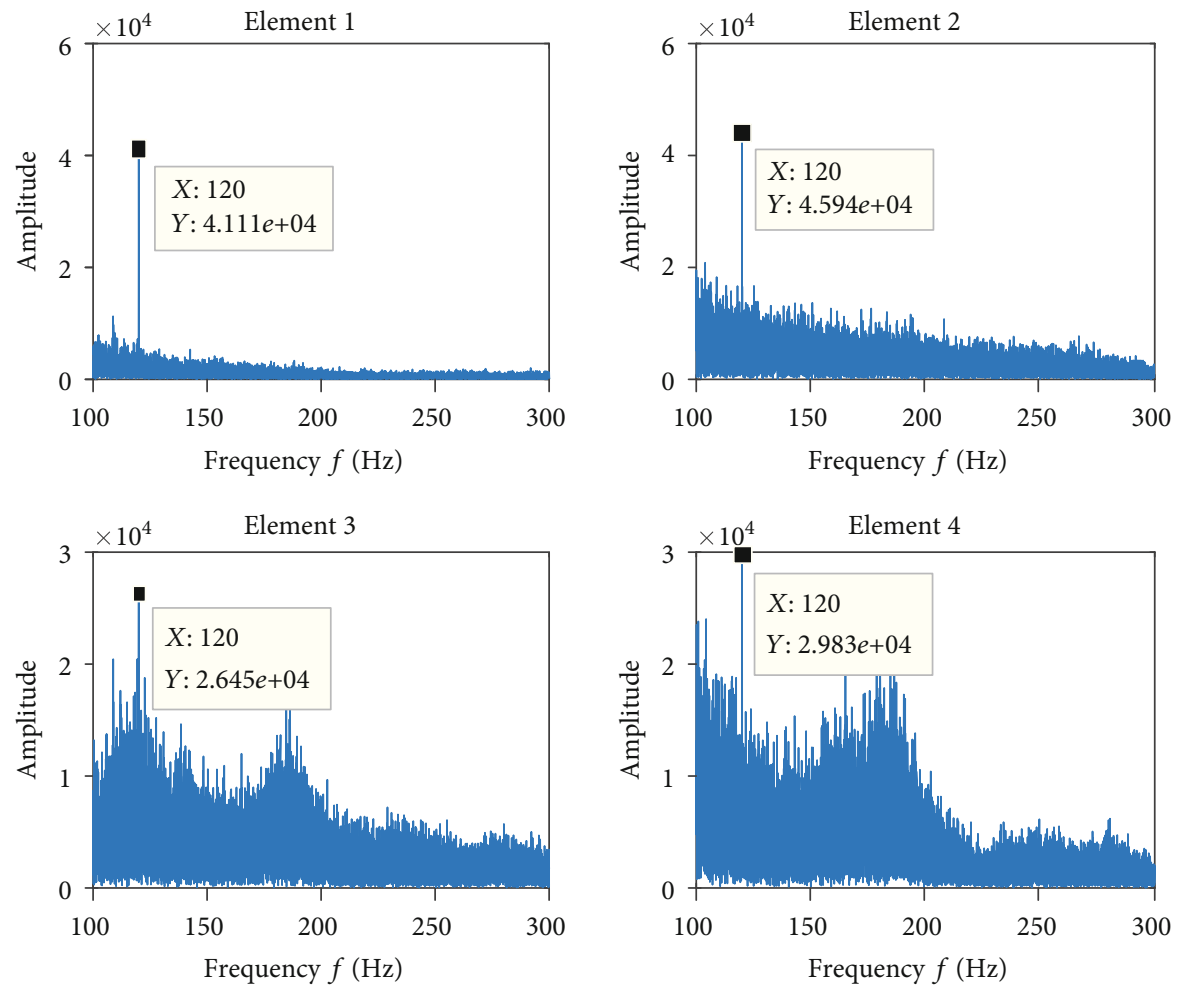

(a)
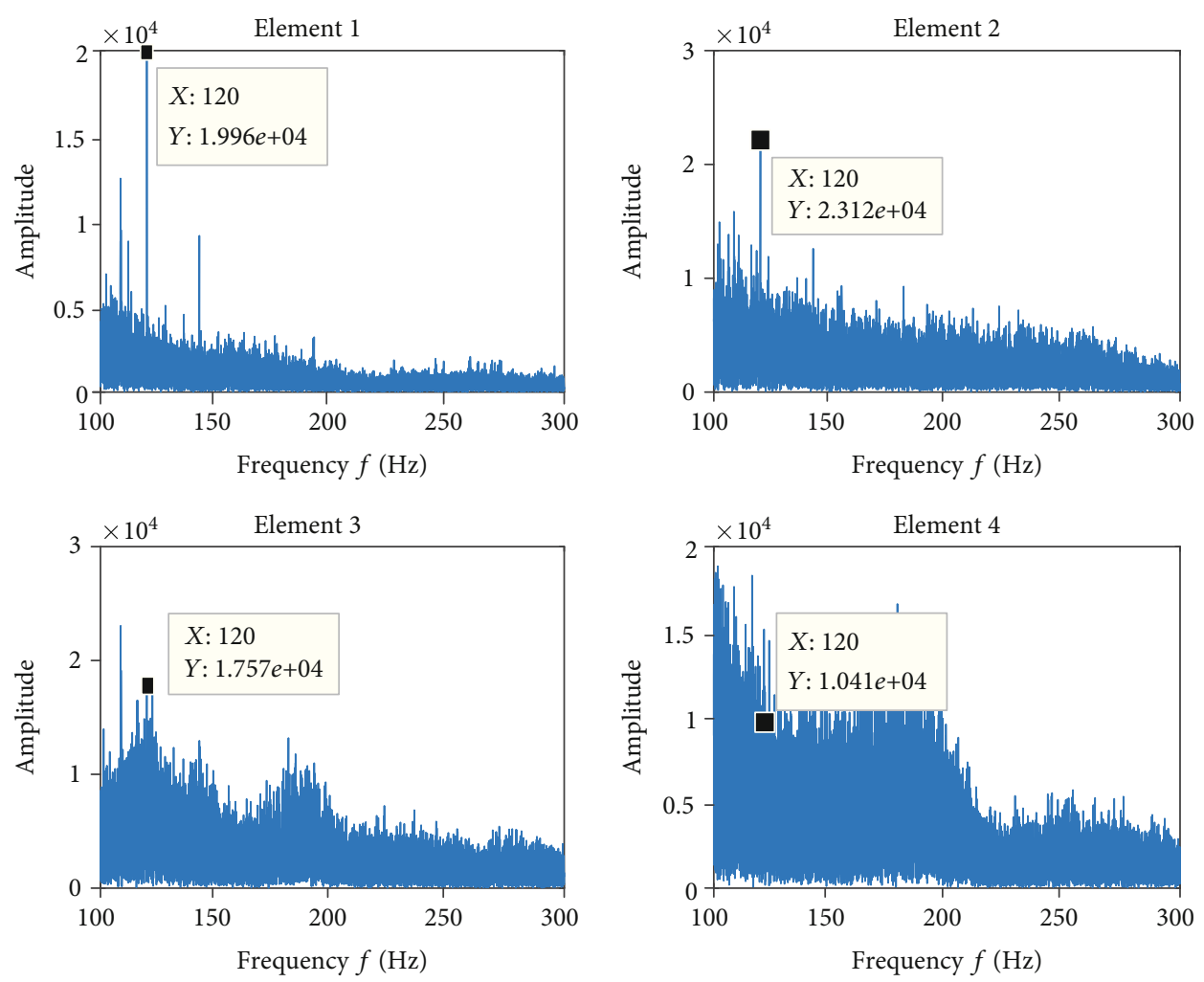

(b)

FIgUre 16: Continued. 

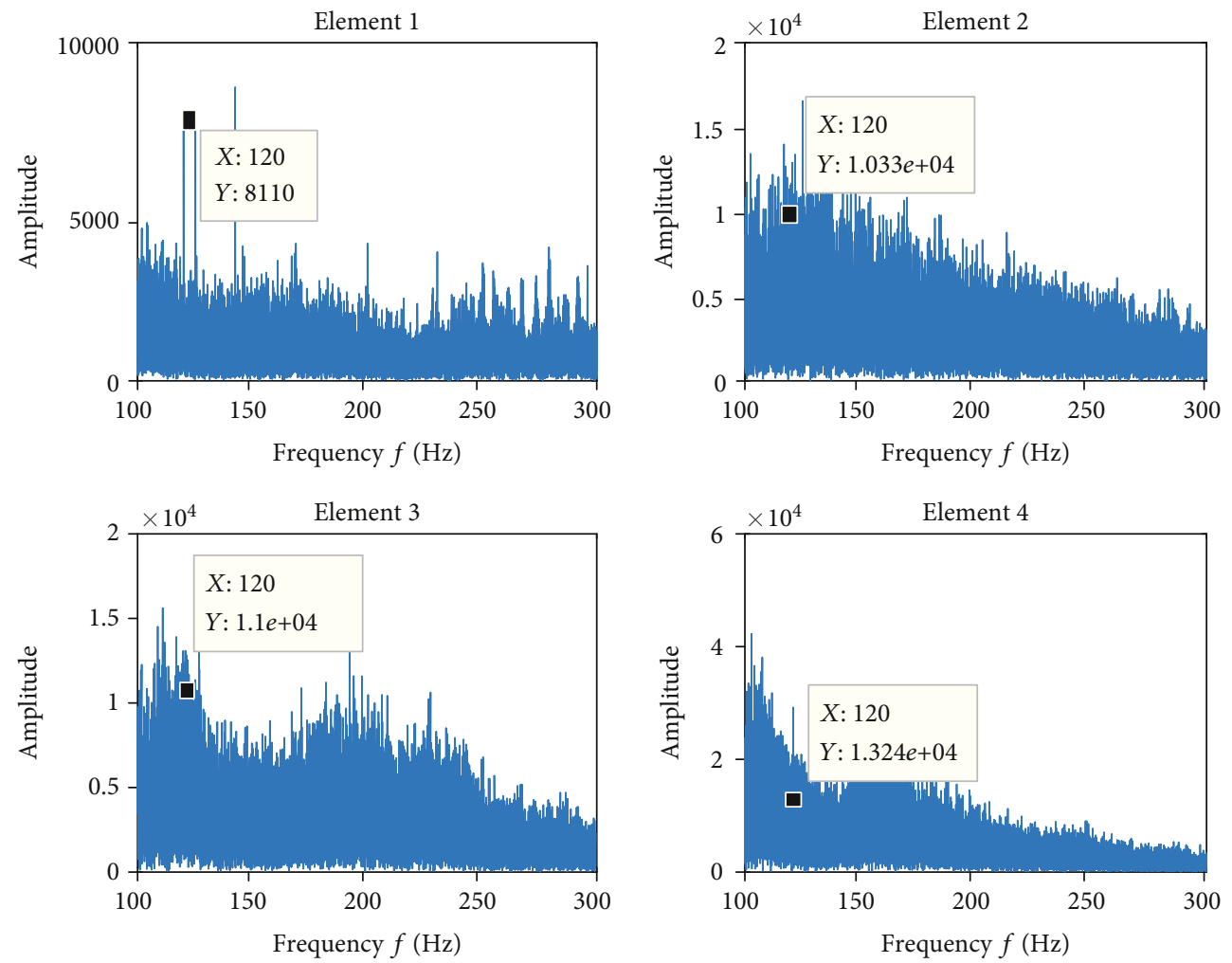

(c)
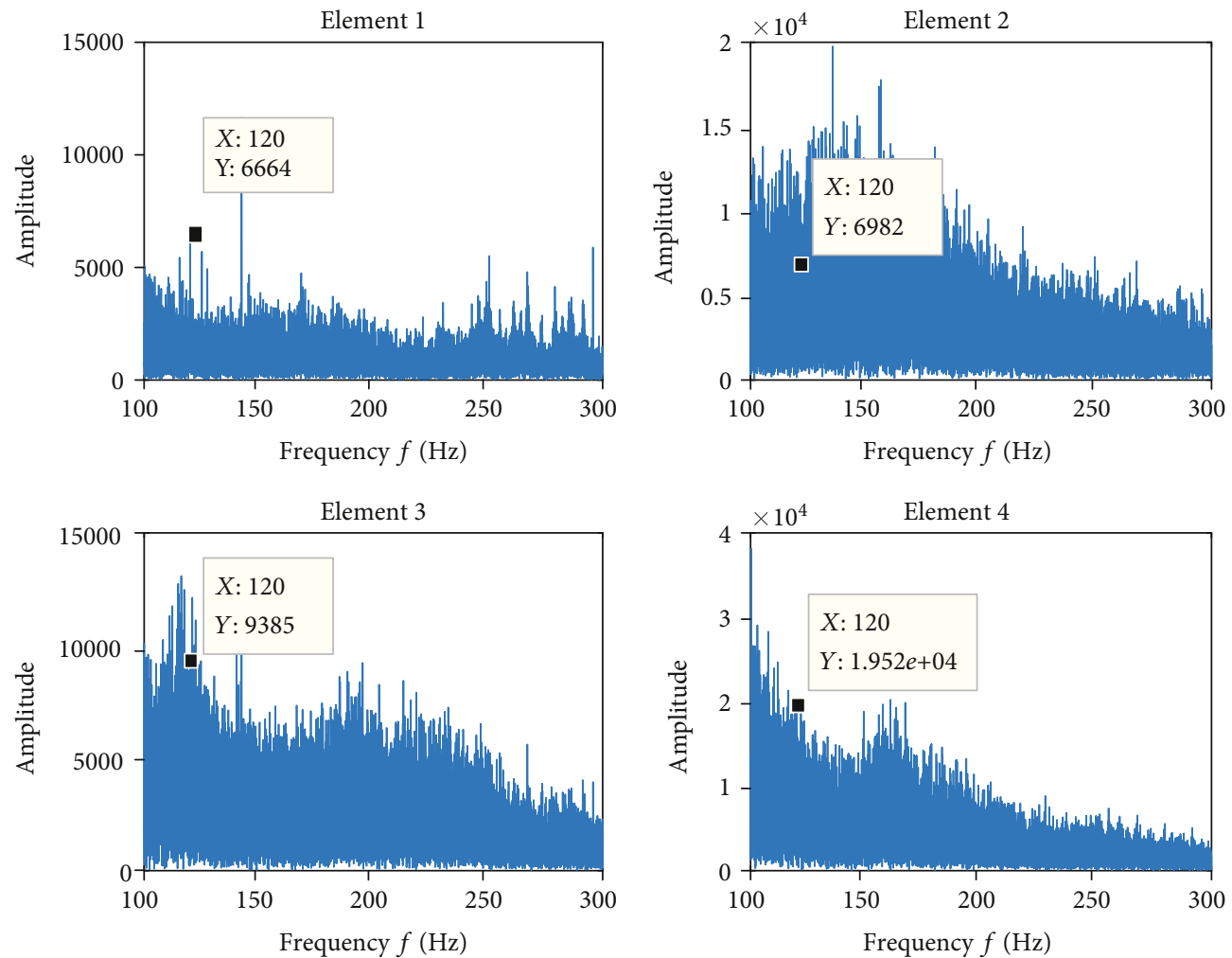

(d)

FIgURE 16: Continued. 

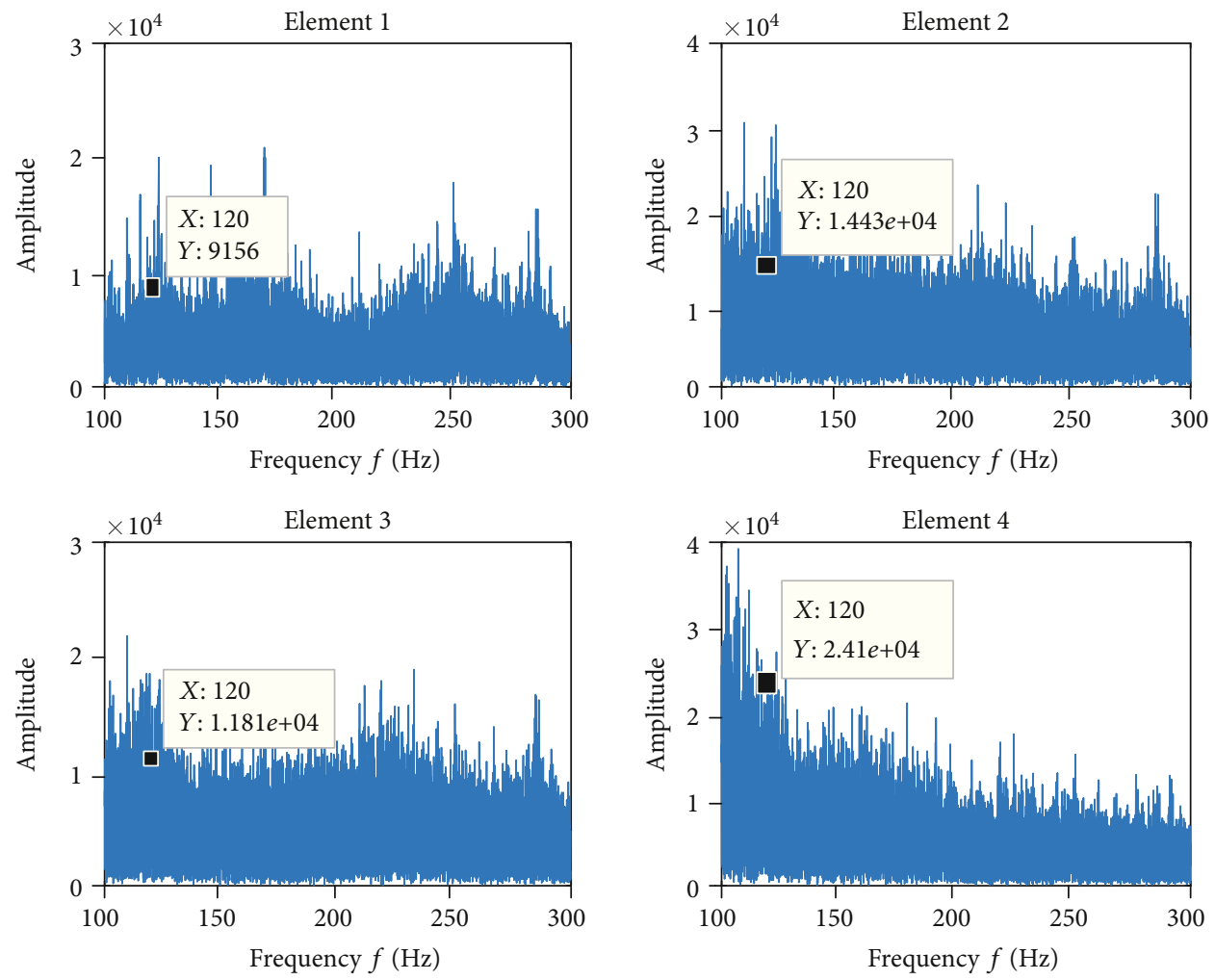

(e)
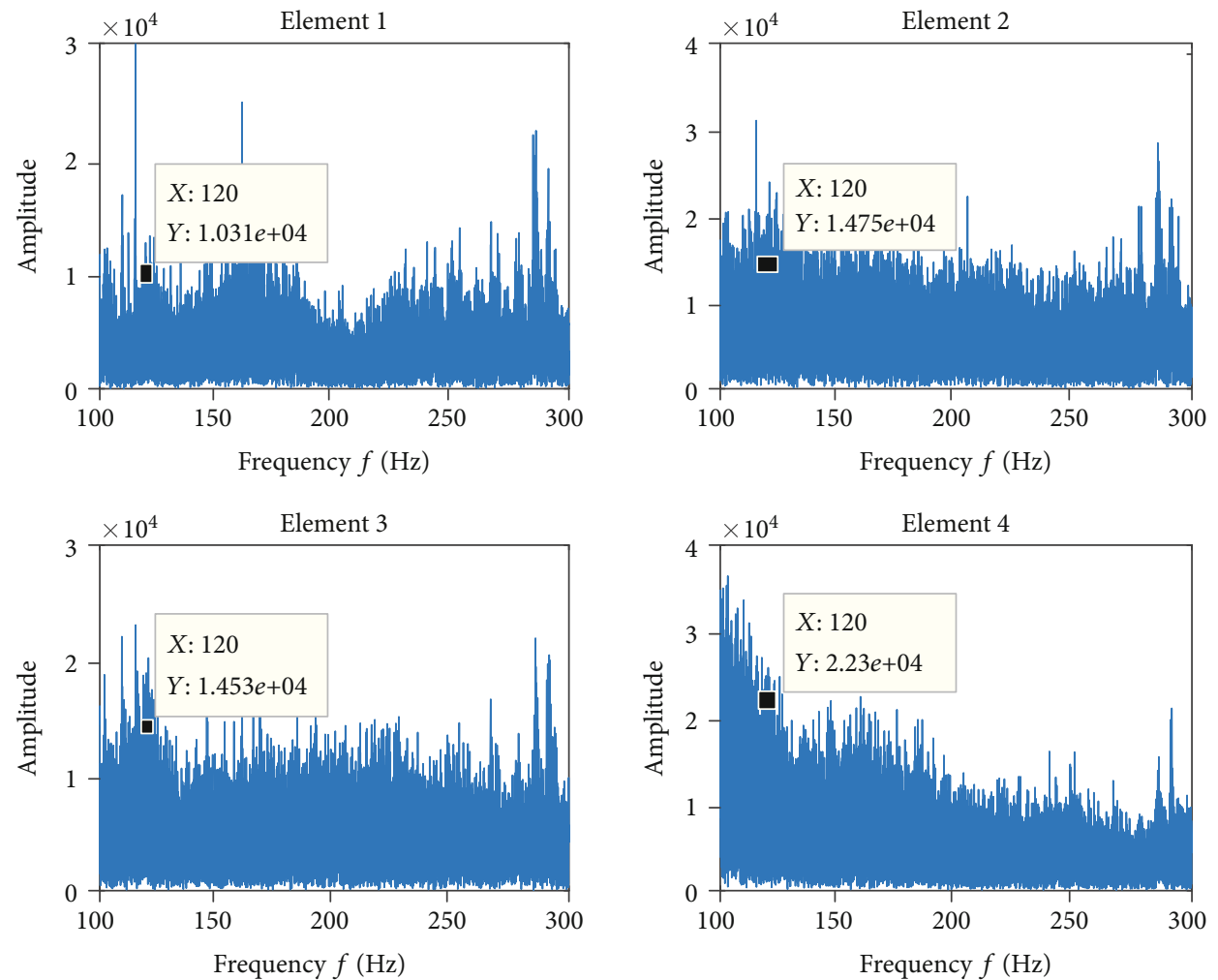

(f)

FIGURE 16: The frequency spectra of the signals collected by four elements. (a) Signal $1(r \approx 3 \mathrm{~km})$. (b) Signal $2(r \approx 3 \mathrm{~km})$. (c) Signal $3(r \approx 5 \mathrm{~km})$. (d) Signal $4(r \approx 5 \mathrm{~km})$. (e) Signal $5(r \approx 7 \mathrm{~km})$. (f) Signal $6(r \approx 7 \mathrm{~km})$. 
TABLe 1: Depth classification accuracy of underwater target.

\begin{tabular}{lcccccc}
\hline Combination number & 1 & 2 & 3 & 4 & 5 & 5 \\
\hline Signal 1 & $99.1 \%$ & $96.5 \%$ & $93.8 \%$ & $89.4 \%$ & $88.5 \%$ & $90.3 \%$ \\
Signal 2 & $86.7 \%$ & $74.3 \%$ & $77.9 \%$ & $69.9 \%$ & $73.5 \%$ & $75.2 \%$ \\
Signal 3 & $57.5 \%$ & $66.4 \%$ & $33.6 \%$ & $57.5 \%$ & $58.4 \%$ & $48.7 \%$ \\
Signal 4 & $60.2 \%$ & $61.1 \%$ & $58.4 \%$ & $61.1 \%$ & $46.0 \%$ & $53.1 \%$ \\
Signal 5 & $77.0 \%$ & $56.6 \%$ & $56.6 \%$ & $41.6 \%$ & $68.1 \%$ & $43.4 \%$ \\
Signal 6 & $73.5 \%$ & $69.9 \%$ & $45.1 \%$ & $62.0 \%$ & $54.9 \%$ & $49.6 \%$ \\
\hline
\end{tabular}

fluctuation than the fifth combination. If the above two combinations can both meet the actual depth classification requirements, it can be considered that the depth classification effect of the third combination is better than that of the fifth combination. The change of the sound velocity profile type will have a certain impact on the depth classification performance, but the impact of the receiving depths on the depth classification performance is similar to that of the isovelocity condition. An improved depth classification effect can be obtained by appropriately adjusting the depths of the two receivers. In addition, according to the simulation results, the source used in this experiment should theoretically be identified as an underwater target.

The PSD has been achieved through smoothed FFT spectrum calculation. The Hamming window is the corresponding weighted window used for smoothing. Its window length and step are $4 \mathrm{~s}$ and $2 \mathrm{~s}$, respectively.

The spectra of the signal 1 to signal 6 received by the four elements in the actual sea experiment are graphed in Figure 16. It can be witnessed from Figure 16 that when the horizontal distance between the source and the receiver is $r$ $\approx 3 \mathrm{~km}$, each spectrum of signal 1 received by four elements has a clear line spectrum at $f=120 \mathrm{~Hz}$, but only the spectra of signal 2 received by the first three elements have a clear line spectrum at $f=120 \mathrm{~Hz}$. There is not a clear line spectrum at $f=120 \mathrm{~Hz}$ in the signal 2 bandwidth received by the fourth element. When $r \approx 5 \mathrm{~km}$, only the spectra of the signals 3 and 4 received by the first element have a clear line spectrum at $f=120 \mathrm{~Hz}$. The spectra of the signals 3 and 4 received by the other three elements do not have a clear line spectrum at $f=120 \mathrm{~Hz}$. When $r \approx 7 \mathrm{~km}$, the spectra of signals 5 and 6 received by all the four elements do not have a clear line spectrum at $f=120 \mathrm{~Hz}$.

Line spectrum intensity and received SNR have a large impact on the depth classification effect. The target depth classification is performed using the signals 1 to 6 , and the probability that the source is accurately discriminated as an underwater target is shown in Table 1. The serial number in the table is the serial number of element combinations.

According to the data in Table 1 , when $r \approx 3 \mathrm{~km}$, the source can be accurately identified as an underwater target using signals collected by each of the six array element combinations. This outcome is identical to the simulation results. When $r \approx 5,7 \mathrm{~km}$, signals collected by only some of the element combinations are useful for target depth classification, and the corresponding classification effects are poor. This is mainly because the source level of the low-frequency source is limited, and the amplification factor of the power amplifier is also limited. So, when the transmitted signal propagates to a distant place the signal becomes weak. The low SNR affects the performance of the target depth classification algorithm. In addition, when $r \approx 3 \mathrm{~km}$, the obtained depth classification results cannot prove the feasibility of the method. This method accurately classifies underwater targets and perfectly classifies surface targets at the same time. In this experiment, only one source depth was tested.

In the actual experiment data processing, there are surface targets such as fishing boats and patrol boats in the sea area. On the contrary, the source carried by the sending ship is the only underwater target which can radiate strong line spectrum signal. Therefore, by performing spectrum analysis on the received signal, if there are clear continuous line spectrum outside the time range of the underwater target radiated signals, they are the spectrum of the signals radiated by surface targets. After time-frequency analysis of the signal in the whole observation time range, it is found that there are evident continuous line spectrum corresponding to the four different segments of received signal as it can be witnessed in Figure 17.

The first segment of signal contains two line spectra whose frequencies are $\left[f_{1}, f_{2}\right]=[106,121.5] \mathrm{Hz}$. The line spectrum frequency of the second signal is $f_{3}=142.5 \mathrm{~Hz}$. There are two line spectra in the third signal, whose frequencies are $\left[f_{4}, f_{5}\right]=[119.8,159.5] \mathrm{Hz} . \quad\left[f_{6}, f_{7}, f_{8}, f_{9}\right]=[123,138.5$, $153.5,169] \mathrm{Hz}$ are line spectrum frequencies of the fourth signal. Each segment of signal containing multiple clear continuous line spectrum may be harmonic signals radiated by the same surface target, but it may also be single or harmonic signals radiated by different surface targets. Therefore, the order of these line spectra is represented by the line spectrum number $f_{1} \sim f_{9}$. The corresponding depth classification results are shown in Table 2 . The data in the table is the probability that the target is accurately classified as the surface target. The serial number in Table 2 is the serial number of the element combinations.

According to the data in Table 2, except for the third line spectrum, only using signals collected by the third and fifth elements can accurately identify the target as a surface target. It is only based on signals collected by these two combinations, whether it is a surface target or an underwater target, its depth can be accurately classified. Among the six combinations, the depth summation of these two combinations is closest to $29 \mathrm{~m}$ exactly, so that the correctness and practical application value of the ideal receiving depth concept have 


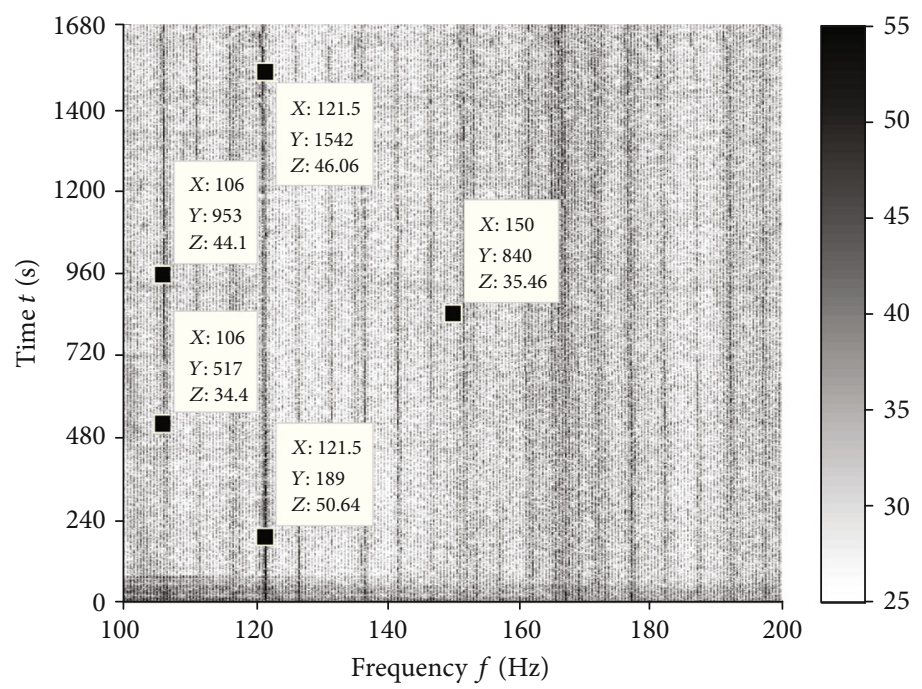

(a)

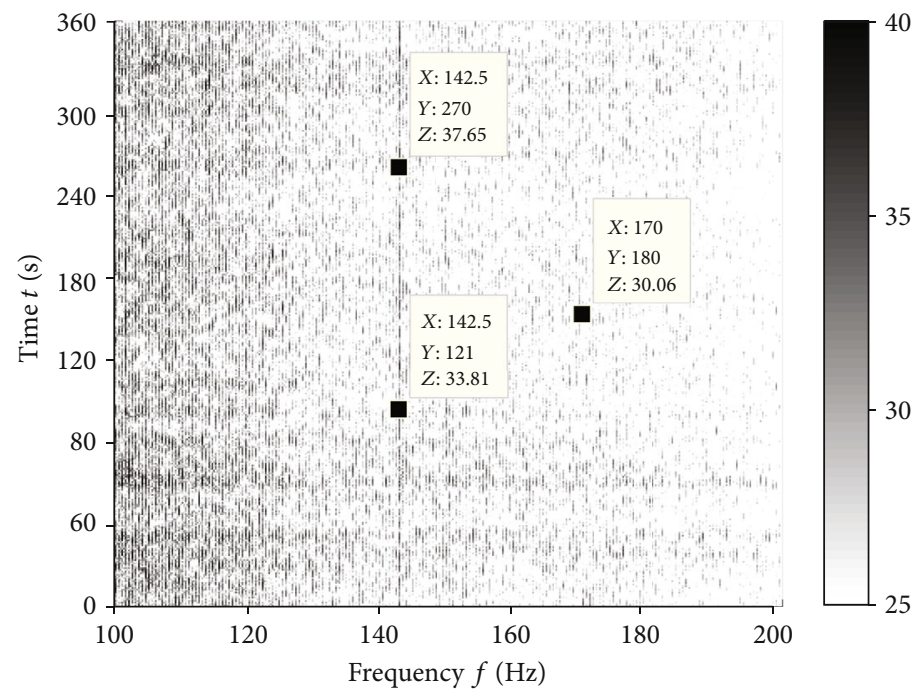

(b)

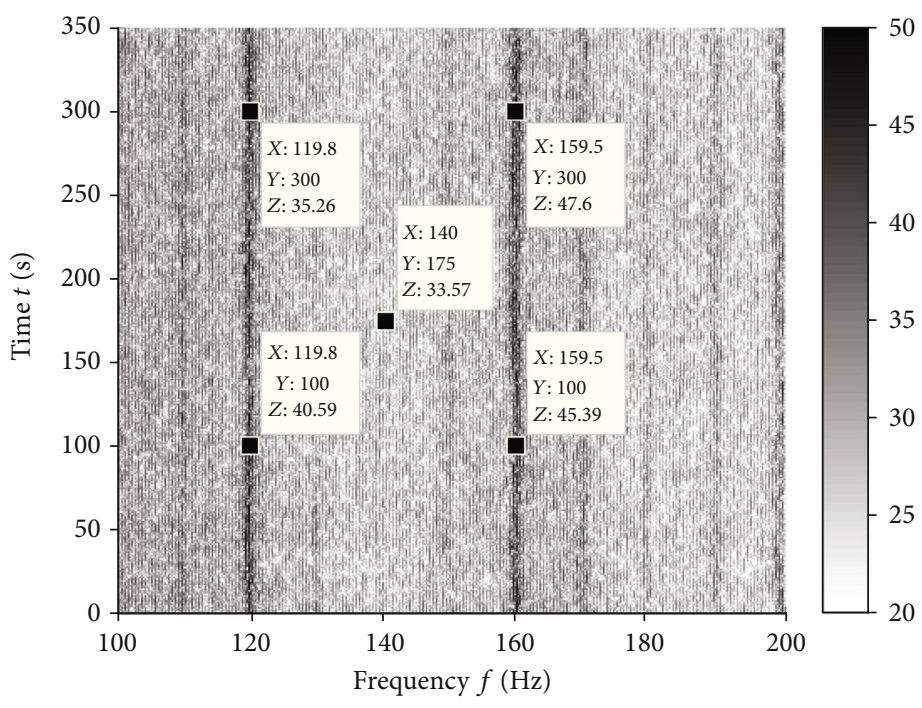

(c)

Figure 17: Continued. 


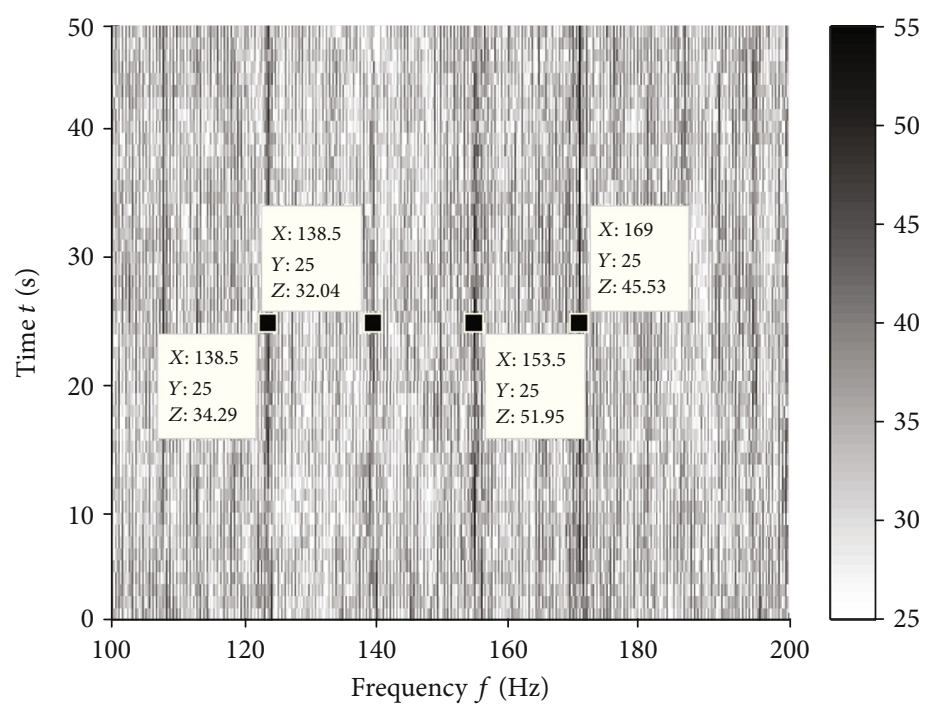

(d)

Figure 17: Time-frequency distributions of surface target radiated signals. (a) First segment of signal. (b) Second segment of signal. (c) Third segment of signal. (d) Fourth segment of signal.

TABLE 2: Depth classification accuracy of surface target.

\begin{tabular}{|c|c|c|c|c|c|c|}
\hline Combination number & 1 & 2 & 3 & 4 & 5 & 6 \\
\hline$f_{1}$ & $13.8 \%$ & $23.5 \%$ & $77.8 \%$ & $14.8 \%$ & $56.8 \%$ & $25.6 \%$ \\
\hline$f_{2}$ & $12.3 \%$ & $21.8 \%$ & $84.3 \%$ & $14.7 \%$ & $68.9 \%$ & $25.2 \%$ \\
\hline$f_{3}$ & $15.6 \%$ & $49.0 \%$ & $46.8 \%$ & $41.4 \%$ & $38.7 \%$ & $40.3 \%$ \\
\hline$f_{4}$ & $2.6 \%$ & $29.8 \%$ & $87.5 \%$ & $15.3 \%$ & $78.2 \%$ & $40.5 \%$ \\
\hline$f_{5}$ & $5.1 \%$ & $89.8 \%$ & $97.5 \%$ & $20.1 \%$ & $86.1 \%$ & $7.7 \%$ \\
\hline$f_{6}$ & $7.1 \%$ & $15.9 \%$ & $81.4 \%$ & $7.1 \%$ & $67.3 \%$ & $30.1 \%$ \\
\hline$f_{7}$ & $16.8 \%$ & $42.5 \%$ & $77.0 \%$ & $21.2 \%$ & $69.9 \%$ & $38.9 \%$ \\
\hline$f_{8}$ & $11.5 \%$ & $86.7 \%$ & $94.7 \%$ & $11.5 \%$ & $76.1 \%$ & $14.2 \%$ \\
\hline$f_{9}$ & $8.0 \%$ & $99.1 \%$ & $97.4 \%$ & $34.5 \%$ & $95.6 \%$ & $0.9 \%$ \\
\hline
\end{tabular}

been verified. In addition, the depth classification effect based on the signals collected by the third element combination is better than the fifth array combination. It is mainly because the depth classification effect is related to the depth summation of the element combination and related to the depth of the first element. Extremely large depth of the first element results in poor classification effect and even it is not able to classify the target depth. Considering the influence of the depth summation of the element combination and the depth of its first element on the performance of the algorithm, the depth classification effect using the third element combination is the best. The above results are consistent with the outcome obtained from the simulation results.

However, using the third line spectrum signal for target depth classification, a good classification effect cannot be obtained because this segment of signal is less continuous than the other received signals, and the received SNR is lower resulting in poor performance of the source depth classification algorithm. So, accurate depth classification results cannot be obtained.
Through the analysis of the data in Table 2 and Figure 17, it can be found that the target depth classification effect is directly related to the line spectrum continuity and the received SNR. The better line spectrum continuity or the larger received signal-to-noise ratio results in a better target depth classification effect.

\section{Conclusions}

This paper analyzes the impact of receiving depth on the performance of the target depth classification algorithm through simulation and sea experiment. After a sufficient theoretical study on the effective depth model, it is verified that when the sensors are at the ideal receiving depth, the performance of the target depth classification algorithm based on the first two modes is significantly better than that of the sensors placed at the nonideal receiving depth. The depth summation of the sensors and the depth value of the first sensor will affect the depth classification effect and critical depth value. The impact of the source frequency on the performance of 
the algorithm has also been researched. The influence of SNR and sound velocity profile parameters (negative gradient, thermocline intensity, thermocline thickness, and upboundary depth) on the presented method has been deeply investigated. Considering the above factors comprehensively, the performance of the depth classification algorithm can be guaranteed to meet the actual requirements by suitably adjusting the receiving depths. The sea experiment was conducted for data processing. The improved performance of the target depth classification algorithm has been verified. The factors affecting the performance of the algorithm in real applications include line spectrum continuity and SNR at the receiver.

There are still some drawbacks and limitations for our presented algorithm in real application scenario. Firstly, the condition of using this algorithm is that source frequency must only excite the first two modes. If the source frequency can excite three or more modes, the algorithm described in this paper is not applicable. This limitation can be overcome by using matched field processing theory in our other study. Secondly, the algorithm has certain requirements for depths of the receivers. The application value and performance of the algorithm will be limited by the actual underwater platform installation requirements. Therefore, according to the installation requirements of the actual underwater platform, it may be necessary to further improve the algorithm through more in-depth research.

\section{Data Availability}

The data used to support the findings of this study were supplied by Nansong Li under license and so cannot be made freely available. Requests for access to these data should be made to Nansong Li (linansong@hrbeu.edu.cn.).

\section{Conflicts of Interest}

The authors declare that there is no conflict of interest regarding the publication of this paper.

\section{Acknowledgments}

This work was supported by the National Natural Science Foundation of China (grant numbers 11374072 and 61371171); the Open Foundation of National Key Laboratory of Science and Technology on Underwater Acoustic Antagonizing (grant number SSDKKFJJ-2018); the Acoustic Science and Technology Laboratory Stable Support Project (grant number SSJSWDZC2018002); the Foundation of Science and Technology on Sonar Laboratory (grant number 614210902011906); and the New Technology Research University Cooperation Project of CETC 54.

\section{References}

[1] S. Golden, "Maximum-likelihood estimation and detection for moving sources in waveguides," in Conference Record of the Thirty-Third Asilomar Conference on Signals, Systems, and Computers (Cat. No.CH37020), Pacific Grove, CA, USA, October 1999.
[2] L. Guo-Long, Z. Yi-Feng, Z. Nan, and W. Jin-Jin, "Matchmode autoregressive method for moving source depth estimation in shallow water waveguides," Mathematical Problems in Engineering, vol. 2018, Article ID 7824671, 15 pages, 2018.

[3] V. E. Premus, J. Ward, and C. D. Richmond, "Mode filtering approaches to acoustic source depth discrimination," in Conference Record of the Thirty-Eighth Asilomar Conference on Signals, Systems and Computers, 2004, Pacific Grove, CA, USA, November 2004.

[4] B. Nicolas, J. I. Mars, and J. L. Lacoume, "Source depth estimation using a horizontal array by matched-mode processing in the frequency-wavenumber domain," EURASIP Journal on Advances in Signal Processing, vol. 2006, no. 1, Article ID 065901, 31 pages, 2006.

[5] T. C. Yang and W. Xu, "Data-based depth estimation of an incoming autonomous underwater vehicle," The Journal of the Acoustical Society of America, vol. 140, no. 4, pp. EL302EL306, 2016.

[6] R. Goldhahn, Waveguide Invariant Active Sonar Target Detection and Depth Classification in Shallow Water, Ph. D. Thesis, Duke University, 2010.

[7] X. Y. Luo, Q. B. Han, D. F. Zhou et al., "Simulation of depth resolution for MFP passive detection," Applied Mechanics and Materials, vol. 742, pp. 136-139, 2015.

[8] A. B. Baggeroer, W. A. Kuperman, and P. N. Mikhalevsky, "An overview of matched field methods in ocean acoustics," IEEE Journal of Oceanic Engineering, vol. 18, no. 4, pp. 401-424, 1993.

[9] Z. H. Michalopoulou and M. B. Porter, "Matched-field processing for broad-band source localization," IEEE Journal of Oceanic Engineering, vol. 21, no. 4, pp. 384-392, 1996.

[10] M. Porter, R. Dicus, and R. Fizell, "Simulations of matchedfield processing in a deep-water pacific environment," IEEE Journal of Oceanic Engineering, vol. 12, no. 1, pp. 173-181, 1987.

[11] E. S. Livingston and H. Schimidt, "A comparison of the conventional, the minimum variance, and the multiple constraint matched field processors," Journal of Computational Acoustics, vol. 2, no. 3, pp. 217-229, 1994.

[12] P. Li, X. H. Zhang, L. F. Fu, and X. X. Zeng, "A modal domain beamforming approach for depth estimation by a horizontal array," Acta Physica Sinica, vol. 66, no. 8, article 084301, 2017.

[13] V.E. Premus and D. Backman, "A matched subspace approach to depth discrimination in a shallow water waveguide," in 2007 Conference Record of the Forty-First Asilomar Conference on Signals, Systems and Computers, Pacific Grove, CA, USA, November 2007.

[14] L. L. Scharf and B. Friedlander, "Matched subspace detectors," IEEE Transactions on Signal Processing, vol. 42, no. 8, pp. 2146-2157, 1994.

[15] L. An, S. L. Fang, and L. J. Chen, "Models for amplitude fluctuation of underwater acoustic narrow band signal based on modified modal scintillation index," Journal of Southeast University, vol. 29, no. 3, pp. 235-241, 2013.

[16] G. Q. Wu, "Estimation of range and depth of a submerged moving object by using noise cepstrum," Journal of Sound and Vibration, vol. 245, no. 5, pp. 835-843, 2001.

[17] L. Zhang, D. Wu, X. Han, and Z. Zhu, "Feature extraction of underwater target signal using mel frequency cepstrum coefficients based on acoustic vector sensor," Journal of Sensors, vol. 2016, Article ID 7864213, 11 pages, 2016. 
[18] S. K. Mitchell and N. R. Bedford, "Determination of source depth from the spectra of small explosions observed at long ranges," The Journal of the Acoustical Society of America, vol. 60, no. 4, pp. 825-828, 1976.

[19] P. Felisberto, O. Rodriguez, P. Santos, E. Ey, and S. M. Jesus, "Experimental results of underwater cooperative source localization using a single acoustic vector sensor," Sensors, vol. 13, no. 7, pp. 8856-8878, 2013.

[20] J. Y. Hui, G. C. Sun, and A. B. Zhao, "Normal mode acoustic intensity flux in Pekeris waveguide and its cross spectra signal processing," Chinese Journal of Acoustics, vol. 28, no. 1, pp. 2127, 2009.

[21] Y. Y. Zhang, Y. Zhang, G. L. Hou, and J. C. Sun, “Acoustic intensity flux in low frequency acoustic field of shallow water and its application research," in Proceedings of 2012 2nd International Conference on Computer Science and Network Technology, Changchun, China, December 2012.

[22] Y. Yu, Q. Ling, and J. Xu, "Pressure and velocity crossspectrum of normal modes in low-frequency acoustic vector field of shallow water and its application," Journal of Systems Engineering and Electronics, vol. 26, no. 2, pp. 241-249, 2015.

[23] G. Yang, J. Yin, Y. Yu, and Z. Shi, "Depth classification of underwater targets based on complex acoustic intensity of normal modes," Journal of Ocean University of China, vol. 15, no. 2, article 2674, pp. 241-246, 2016.

[24] X. T. Wu, Y. Huang, S. G. Gao, and C. H. Zhang, "Source depth discrimination based on double hydrophones," Journal of Applied Acoustics, vol. 35, no. 2, pp. 179-188, 2016.

[25] L. Zhang, Y. Huang, W. J. Li, S. G. Gao, J. Tian, and X. Wu, "Depth estimation of objects with vector vertical array," Journal of Signal Processing, vol. 32, no. 4, pp. 388-394, 2016.

[26] R. N. Denham, "Asymptotic solution for the sound field in shallow water with a negative velocity gradient," The Journal of the Acoustical Society of America, vol. 45, no. 2, pp. 365371, 1969.

[27] M. J. Buckingham and E. M. Giddens, "On the acoustic field in a Pekeris waveguide with attenuation in the bottom halfspace," The Journal of the Acoustical Society of America, vol. 119, no. 1, pp. 123-142, 2006. 


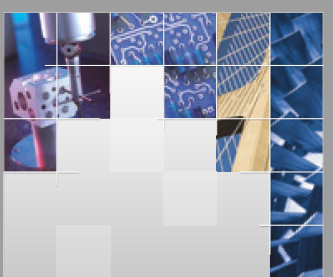

\section{Enfincering}
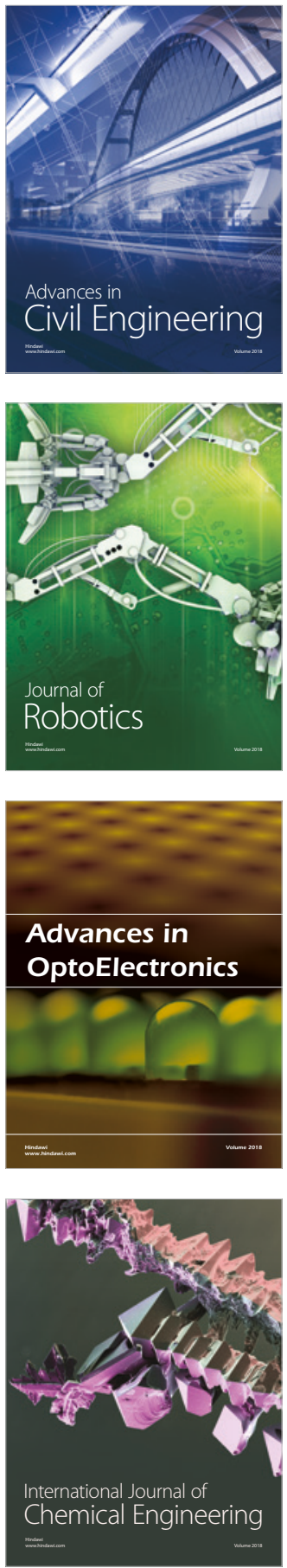

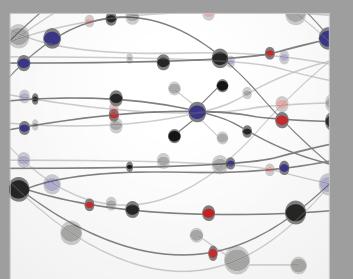

\section{Rotating \\ Machinery}

The Scientific World Journal

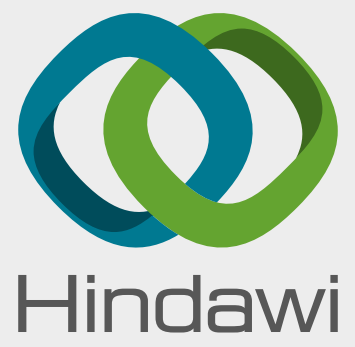

Submit your manuscripts at

www.hindawi.com
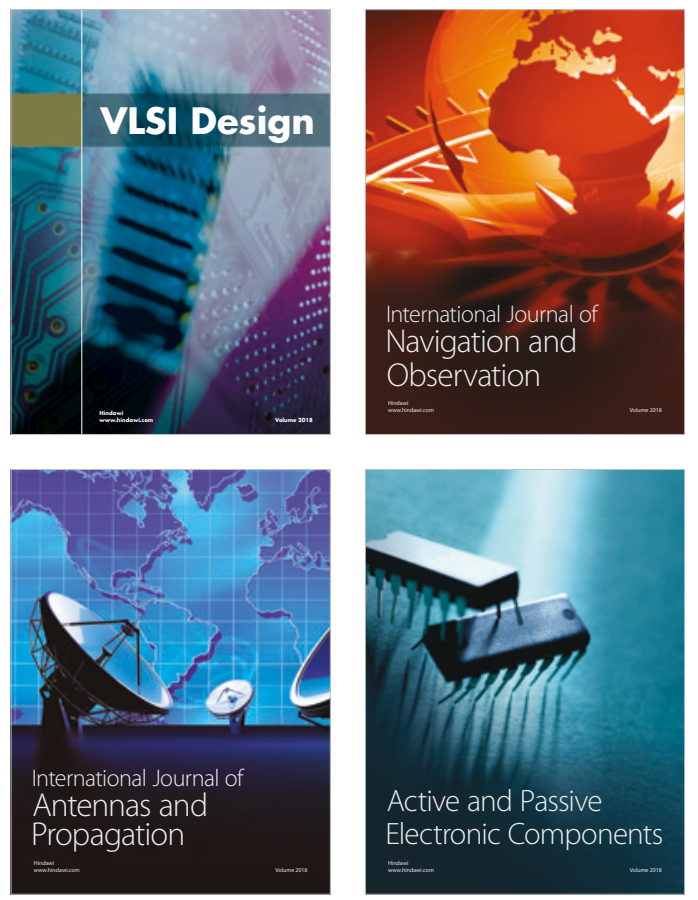
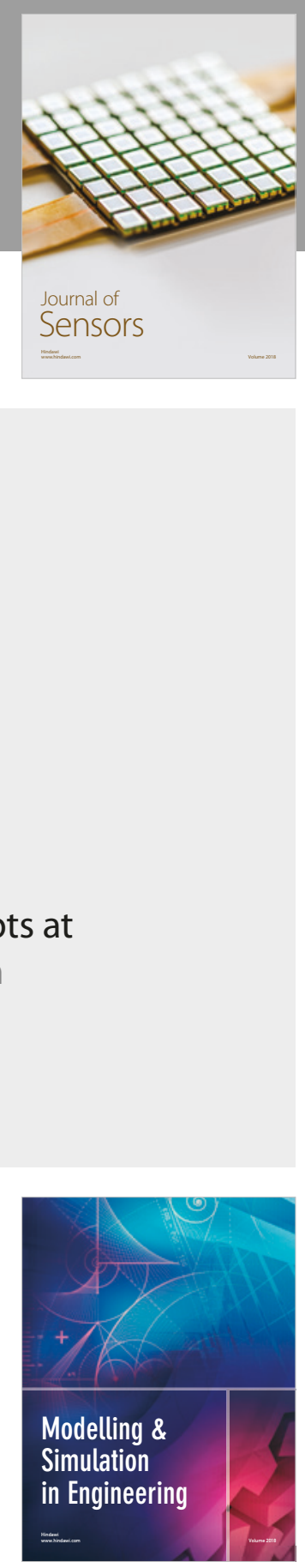

\section{Advances \\ Multimedia}
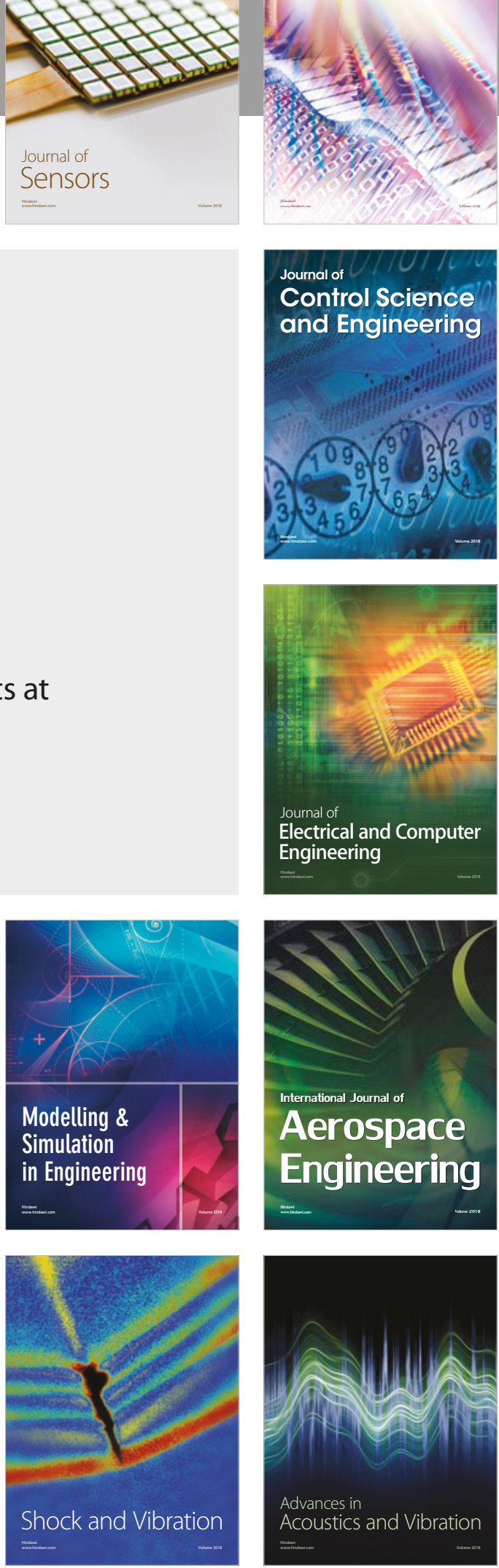